\title{
Efficient Synthesis of Room Acoustics via Scattering Delay Networks
}

\author{
Enzo De Sena, Member, IEEE, Hüseyin Hacihabiboğlu, Senior Member, IEEE, \\ Zoran Cvetković, Senior Member, IEEE, Julius O. Smith, Member, IEEE
}

\begin{abstract}
An acoustic reverberator consisting of a network of delay lines connected via scattering junctions is proposed. All parameters of the reverberator are derived from physical properties of the enclosure it simulates. It allows for simulation of unequal and frequency-dependent wall absorption, as well as directional sources and microphones. The reverberator renders the first-order reflections exactly, while making progressively coarser approximations of higher-order reflections. The rate of energy decay is close to that obtained with the image method (IM) and consistent with the predictions of Sabine and Eyring equations. The time evolution of the normalized echo density, which was previously shown to be correlated with the perceived texture of reverberation, is also close to that of IM. However, its computational complexity is one to two orders of magnitude lower, comparable to the computational complexity of a feedback delay network (FDN), and its memory requirements are negligible.
\end{abstract}

Index Terms-Room acoustics, acoustic simulation, digital waveguide network, reverberation time, echo density.

\section{INTRODUCTION}

A comprehensive account of the first fifty years of artificial reverberation in [1] identifies three main classes of digital reverberators: delay networks, physical room models

Copyright (C) 2015 IEEE. Personal use of this material is permitted. However, permission to use this material for any other purposes must be obtained from the IEEE by sending a request to pubs-permissions@ @ieee.org. http://ieeexplore.ieee.org/xpl/articleDetails.jsp?arnumber=7113826

Manuscript received February 19, 2015; accepted May 16, 2015. Date of publication June 01, 2015. The work reported in this paper was partially funded by (i) EPSRC Grant EP/F001142/1, (ii) European Commission under Grant Agreement no. 316969 within the FP7-PEOPLE Marie Curie Initial Training Network "Dereverberation and Reverberation of Audio, Music, and Speech (DREAMS)", and (iii) TUBITAK Grant 113E513 "Spatial Audio Reproduction Using Analysis-based Synthesis Methods". The method presented in this paper is protected by USPTO Patent $n$. 8908875. The associate editor coordinating the review of this manuscript and approving it for publication was Prof. Bozena Kostek.

Enzo De Sena is with ESAT-STADIUS, KU Leuven, Kasteelpark Arenberg 10, 3001 Leuven, Belgium (e-mail: enzo.desena@esat.kuleuven.be). This work was done in part while he was with CTR, King's College London.

Zoran Cvetković is with the Centre for Telecommunications Research (CTR), King's College London, Strand, London, WC2R 2LS, United Kingdom (e-mail: zoran.cvetkovic@kcl.ac.uk).

Hüseyin Hacıhabiboğlu is with Department of Modeling and Simulation, Informatics Institute, Middle East Technical University, Ankara, 06800, Turkey (e-mail: hhuseyin@metu.edu.tr). This work was done in part while he was with King's College London.

Julius O. Smith III is with CCRMA, Stanford University, Stanford, CA 94304, USA.

This paper has supplementary downloadable material available at http://ieeexplore.ieee.org provided by the author. The material includes several audio samples generated using the proposed room acoustic simulator. Contact enzo.desena@esat.kulueven.be for further questions about this work. and convolution-based algorithms. The earliest class consisted of delay networks, which were the only artificial reverberators feasible with the integrated circuits of the time. The first delay network reverberator, as introduced by Schroeder, was a cascade of several allpass filters, a parallel bank of feedback comb filters and a mixing matrix [1]-[3]. Since then, a large number of delay networks have been proposed and used commercially. Most of these networks were designed heuristically and by trial and error. Feedback delay networks (FDNs) were developed on a more solid scientific grounding as a multichannel extension of the Schroeder reverberator [4], [5], and consist of parallel delay lines connected recursively through a unitary feedback matrix. The state-of-the-art FDN is due to Jot and Chaigne [6], who proposed using delay lines connected in series with multiband absorptive filters to obtain a frequency-dependent reverberation time. FDN reverberators are still among the most commonly used artificial reverberators owing to their simple design, extremely low computational complexity and high reverberation quality. While no new standard seems to have emerged yet, a number of more intricate networks have been proposed more recently that show improvements over FDNs, sometimes even in terms of computational complexity [7]-[9].

FDNs are structurally equivalent to a particular case of digital waveguide networks (DWNs) [10], reverberators based on the concept of digital waveguides, introduced in [11]. FDNs and DWNs can also be viewed both as networks of multiport elements, as explained by Koontz in [12]. DWNs consist of a closed network of bidirectional delay lines interconnected at lossless junctions. DWNs have an exact physical interpretation as a network of interconnected acoustical tubes and have a number of appealing properties in terms of computational efficiency and numerical stability. Reverberators based on delay networks have been widely used for artistic purposes in music production. High-level interfaces enable artists and sound engineers to adjust the available free parameters until the intended qualities of reverberated sound are achieved.

In the domain of predictive architectural modeling, virtual reality and computer games, on the other hand, the objective of artificial reverberators is to emulate the response of a physical room given a set of physically relevant parameters [13], [14]. These parameters include, for instance, the room geometry, absorption and diffusion characteristics of walls and objects, and position and directivity pattern of source and microphone.

Various physical models have been proposed in the past for the purpose of room acoustic synthesis. Widely used geometric-acoustic models make the simplifying assumption 
that sound waves propagate as rays. In particular, the ray tracing approach explicitly tracks the rays emitted by the acoustic source as they bounce off the surfaces of a modeled enclosure. The image method (IM) is an alternative algorithmic implementation that formally replaces the physical boundaries surrounding the source with an equivalent infinite lattice of image sources [15], [16]. Allen and Berkley proved that, in the case of rectangular rooms, this approach is equivalent to solving the wave equation provided that the walls are perfectly rigid [15]. When the walls are not rigid, the results of the IM are no longer physically accurate, but the method still retains its geometric-acoustic interpretation. The IM can also be used to model the acoustics in arbitrary polyhedra, as described by Borish in [16]. The main advantage of geometric-acoustic models in comparison to other physical models is their lower - although still considerable - computational complexity. However, they do not model important wave phenomena such as diffraction and interference. These phenomena are inherently modeled by methods based on time and space discretization of the wave equation, such as finite-difference time-domain (FDTD) and digital waveguide mesh (DWM) models [14], [17]-[19]. The main limitation of physical room models consists of their significant computational and memory requirements. While this may not be problematic for predictive acoustic modeling applications, it is a significant limitation for interactive applications, such as virtual reality (VR). Similarly, convolutional methods, which operate by filtering anechoic audio samples with measured room impulse responses (RIRs), do not allow interactive operation unless interpolation among an extensive number of RIRs is supported.

Virtual reality has become a widespread technology, with applications in military training, immersive virtual musical instruments, archaeological acoustics, and, in particular, computer games. Along with realistic graphics rendering, spatial audio is one of the most important factors that affect how convincing its users perceive a virtual environment [20]. The aural and visual components should be consistent and mutually supportive so as to minimise cross-modal sensory conflicts [20].

Room acoustic synthesis for VR also requires flexibility in terms of audio output devices. For example, a full scale VR suite such as a CAVE automatic virtual environment (CAVE) can use Ambisonics [21] or wave field synthesis (WFS), which requires from tens to hundreds of loudspeaker channels [22]. In contrast, a portable game console has a two-channel output that may be used, for instance, to reproduce binaural audio over headphones [23]. Typical home users, on the other hand, commonly use stereophonic, 5.1 or 7.1 setups, whereas the ultra high definition TV (UHDTV) standard, also aimed at home users, makes provisions for 22.2 setups [24].

In summary, room acoustic synthesizers for VR require (i) explicit (tuning-free) modeling of a given virtual space, (ii) scalability in terms of playback configuration, and (iii) low computational complexity. On the other hand, of the three classes of digital reverberators, (i) convolutional methods do not allow interactive operation without extensive tabulation and interpolation, (ii) delay network methods do not model explicitly a given virtual space, and (iii) physical models have a high computational cost.

In order to combine the appealing properties of delay networks and physical models, one possible approach consists of designing delay networks that have parameters with an explicit physical interpretation. Studies in [10], [25] and [26] follow this direction. In [10] and [25] the length of the delay lines of FDNs are chosen such that the lowest eigenfrequencies of the room are reconstructed exactly. In [26], Karjalainen et al. use a DWN with few junctions to model a rectangular room. The rationale behind the design is to aggressively prune-down a DWM in order to reduce the complexity while retaining an acceptable perceptual result. This approach has various advantages but requires careful manual tuning in order to provide satisfactory results [26].

In [27] and [28], following the same concept of DWN structures as studied by Karjalainen et al. in [26], we presented an architecture that has a number of appealing properties. The proposed architecture, which we refer to as scattering delay network (SDN), renders the direct-path component and firstorder early reflections accurately both in time and amplitude, while producing a progressively coarser approximation of higher-order reflections. For this reason, SDN can be interpreted as an approximation to geometric acoustics models [15], [16]. SDNs thus approach the accuracy of full-scale room simulation while maintaining computational efficiency on par with typical delay network-based methods. Furthermore, the parameters of SDN are inherited directly from room geometry and absorption properties of wall materials, and therefore do not require ad hoc tuning.

This paper further explores and completes the design presented in [27] and [28]. All design choices are now explained on a physical basis. Furthermore, the paper includes a theoretical analysis of optimal scattering matrices, a comparison with the IM in terms of reverberation time and normalized echo density [29], an analysis of the computational complexity and of the memory requirements, and an analysis of the modal density [30]. The paper is organized as follows. Section II presents a brief overview of FDNs, DWNs and models proposed by Karjalainen et al. [26]. Section III] describes the proposed SDN method. The properties of SDNs are studied in Section IV. Section $\mathrm{V}$ presents numerical evaluation results. Section VI concludes the paper.

\section{BACKGROUND}

The proposed SDN reverberator draws inspiration from DWN and DWM structures, however it is in essence a recursive linear time-invariant system. Hence, to provide a comprehensive context, in this section we briefly review FDNs, which are the most commonly used recursive linear timeinvariant reverberators, followed by a more detailed review of relevant DWN and DWM material.

\section{A. Feedback Delay Networks}

The canonical feedback delay network (FDN) form, as proposed by Jot and Chaigne [6], is shown in Fig. 1] Here, $\mathbf{b}$ and $\mathbf{c}$ are input and output gains, respectively, $\mathbf{D}(z)=\operatorname{diag}\left(z^{-m_{1}}, z^{-m_{2}}, \ldots, z^{-m_{N}}\right)$ are integer delays, 


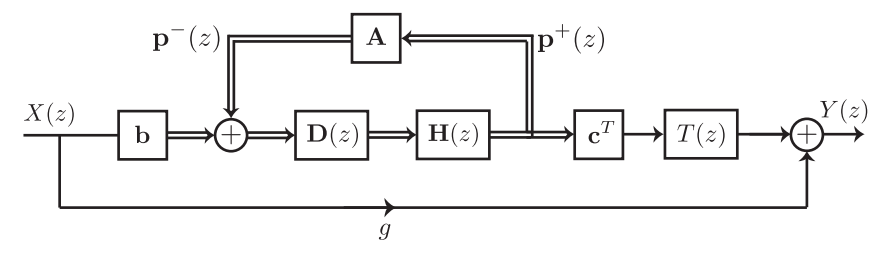

Fig. 1. Block diagram of the modified FDN reverberator as proposed by Jot and Chaigne [6].

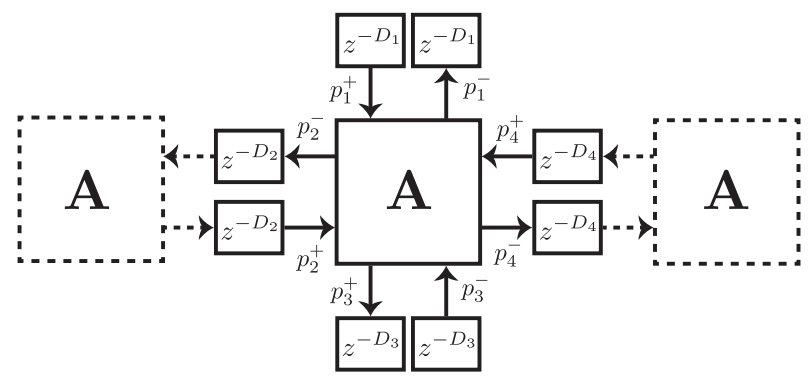

Fig. 2. Operation of a DWN around a junction with $K=4$ waveguides.

$\mathbf{H}(z)=\operatorname{diag}\left(H_{1}(z), \ldots, H_{N}(z)\right)$ are absorption filters, $T(z)$ is the tone correction filter, $g$ is the gain of the direct path, and $\mathbf{A}$ is the feedback matrix. The absorption filters can be designed so as to obtain a desired reverberation time in different frequency bands [6], or to match those calculated from a measured RIR [31]. To achieve a high-quality late reverberation, the feedback loop should be lossless, i.e. energypreserving, hence typically the feedback matrix $\mathbf{A}$ is designed to be unitary. Each particular choice of the feedback matrix has corresponding implications on subjective or objective qualities of the reverberator [32]; e.g. in the particular case of the identity matrix, $\mathbf{A}=\mathbf{I}$, the FDN structure reduces to $N$ comb filters connected in parallel and acts as the Schroeder reverberator [3]. Note, however that unitary matrices are only a subset of possible lossless feedback matrices [10], [33]; we elaborate on this point in the next subsection.

\section{B. Digital Waveguide Networks}

DWNs consist of a closed network of digital waveguides [11]. A digital waveguide is made up of a pair of delay lines, which implement the digital equivalent of the d'Alembert solution of the wave equation in a one-dimensional medium. The digital waveguides are interconnected at junctions, characterised by corresponding scattering matrices. Fig. 2 shows an example of four digital waveguides with length $D_{1}, \ldots, D_{4}$ samples that meet at a junction with scattering matrix A. In general, a junction scatters incoming wave variables $\mathbf{p}^{+}=\left[p_{1}^{+}, \ldots, p_{K}^{+}\right]_{T}^{T}$ to produce outgoing wave variables $\mathbf{p}^{-}=\left[p_{1}^{-}, \ldots, p_{K}^{-}\right]^{T}$ according to $\mathbf{p}^{-}=\mathbf{A} \mathbf{p}^{+}$. Note that if all digital waveguides are terminated by an ideal non-inverting reflection, the DWN is structurally equivalent to the feedback loop of an FDN with feedback matrix $\mathbf{A}$ and delay-line lengths of $2 D_{1}, \ldots, 2 D_{4}$ samples [10].

DWN junctions are lossless. In this context, losslessness is defined according to classical network theory [34]. In particular, a junction with scattering matrix $\mathbf{A}$ is said to be lossless if the input and output total complex power are equal:

$$
\mathbf{p}^{+*} \mathbf{Y} \mathbf{p}^{+}=\mathbf{p}^{-*} \mathbf{Y} \mathbf{p}^{-} \Rightarrow \mathbf{A}^{*} \mathbf{Y A}=\mathbf{Y}
$$

where $\mathbf{Y}$ is a Hermitian positive-definite matrix [10] and $(\cdot)^{*}$ denotes the conjugate transpose. The quantity $\mathbf{p}^{ \pm *} \mathbf{Y} \mathbf{p}^{ \pm}$ is the square of the elliptic norm of $\mathbf{p}^{ \pm}$induced by $\mathbf{Y}$. It can be shown that a matrix $\mathbf{A}$ is lossless if and only if its eigenvalues lie on the unit circle and it admits a basis of linearly independent vectors [10]. A consequence of this result is that lossless feedback matrices can be fully parametrized as $\mathbf{A}=\mathbf{T}^{-1} \boldsymbol{\Lambda} \mathbf{T}$, where $\mathbf{T}$ is any invertible matrix and $\boldsymbol{\Lambda}$ is any unit-modulus diagonal matrix [10].

The DWN can also be interpreted as a physical model for a network of acoustic tubes. In this case $\mathbf{A}$ assumes a particular form. If we denote by $y_{i}$ the characteristic admittance of the $i$-th tube and by $v_{i}$ the volume velocity of the $i$-th tube at the junction, the continuity of pressure and conservation of velocity at the junction give, from [34]:

$$
\begin{aligned}
& p_{1}=p_{2}=\cdots=p_{K}=p, \\
& v_{1}+v_{2}+\cdots+v_{K}=0,
\end{aligned}
$$

respectively, where $p_{i}=p_{i}^{+}+p_{i}^{-}$denotes the acoustic pressure of the $i$-th tube. Equations (2) and (3) imply that the pressure $p$ at the junction is given by

$$
p=\frac{2}{\sum_{i=1}^{K} y_{i}} \sum_{i=1}^{K} y_{i} p_{i}^{+}=\frac{2}{\sum_{i=1}^{K} y_{i}} \sum_{i=1}^{K} y_{i} p_{i}^{-},
$$

where we used $v_{i}=v_{i}^{+}+v_{i}^{-}$and Ohm's law for traveling waves $v_{i}^{+}=y_{i} p_{i}^{+}$and $v_{i}^{-}=-y_{i} p_{i}^{-}$[34]. Since $p_{i}^{-}=p-p_{i}^{+}$, the scattering matrix can be expressed as

$$
\mathbf{A}=\frac{2}{\langle\mathbf{1}, \mathbf{y}\rangle} \mathbf{1 y}^{T}-\mathbf{I},
$$

where $\mathbf{1}=[1, \ldots, 1]^{T}, \mathbf{y}=\left[y_{1}, \ldots, y_{K}\right]^{T},\langle\cdot, \cdot\rangle$ denotes the scalar product, and $\mathbf{I}$ is the identity matrix. Observe that the scattering matrix in (5) satisfies equation (1) with $\mathbf{Y}=\operatorname{diag}\left\{y_{1}, \ldots, y_{K}\right\}$ and is therefore lossless. In this physically-based case, the square of the elliptic norm of $\mathbf{p}^{ \pm}$ induced by $\mathbf{Y}$ has the meaning of incoming/outgoing acoustic power: $\mathbf{p}^{ \pm *} \mathbf{Y} \mathbf{p}^{ \pm}=\sum_{i=1}^{K} y_{i}\left|p_{i}^{ \pm}\right|^{2}= \pm \sum_{i=1}^{K} v_{i}^{*} p_{i}^{ \pm}[10]$.

An equivalent formulation of DWNs involves normalized pressure waves, defined as $\widetilde{p}_{i}^{ \pm}=p_{i}^{ \pm} \sqrt{y_{i}}$. In this case, the propagating wave variables $\widetilde{p}_{i}^{ \pm}$represent the square root of the traveling signal power [10]. If we define $\widetilde{\mathbf{Y}}=$ $\operatorname{diag}\left(\sqrt{y_{1}}, \ldots, \sqrt{y_{K}}\right)$, the normalized output wave can be written as $\widetilde{\mathbf{p}}^{-}=\widetilde{\mathbf{Y}} \mathbf{p}^{-}=\widetilde{\mathbf{Y}} \mathbf{A} \mathbf{p}^{+}=\widetilde{\mathbf{Y}} \mathbf{A} \widetilde{\mathbf{Y}}^{-1} \widetilde{\mathbf{p}}^{+}=\widetilde{\mathbf{A}} \widetilde{\mathbf{p}}^{+}$, where $\widetilde{\mathbf{A}}=\widetilde{\mathbf{Y}} \mathbf{A} \widetilde{\mathbf{Y}}^{-1}$. The equivalent scattering matrix $\widetilde{\mathbf{A}}$ can be expressed as

$$
\widetilde{\mathbf{A}}=\frac{2}{\|\widetilde{\mathbf{y}}\|^{2}} \widetilde{\mathbf{y}} \widetilde{\mathbf{y}}^{T}-\mathbf{I}
$$

which is a Householder reflection around the vector $\widetilde{\mathbf{y}}=$ $\left[\sqrt{y_{1}}, \ldots, \sqrt{y_{K}}\right]^{T}$. Such Householder matrices will be also used in the context of SDN reverberators, proposed in the next section, where they will exhibit some sought-after properties, including low computational complexity and desirable normalised echo density profiles. 
In order to inject energy in a DWN, various methods have been used in the past, ranging from attaching an additional waveguide where the outgoing wave is ignored [11] or using an adapted impedance such that there is no energy reflected along the outgoing wave, to more complex approaches [35]. A common approach is to apply an external ideal volume velocity source to the junction [19]. This is equivalent to superimposing source pressure, $p_{S}$, to the pressure due to the waveguides meeting at the junction, $p$, thus making the total pressure at the junction equal to:

$$
\bar{p}=p_{S}+p
$$

In the context of FDTD models, a source that injects energy in this way is called a soft-source, as opposed to a hardsource, which actively interferes with the propagating pressure field [36], [37]. In order to implement equation (7) in the DWN structure, the input from the source can be distributed uniformly to incoming wave variables according to

$$
\overline{\mathbf{p}}^{+}=\mathbf{p}^{+}+\frac{p_{S}}{2} \mathbf{1}
$$

which provides the intended node pressure [38]. This is the approach that will be used for injecting source energy in the proposed SDN structures.

\section{Digital Waveguide Meshes}

DWNs formed of fine grids of scattering junctions, referred to as digital waveguide meshes, are used to model wave propagation in an acoustic medium [39]. Each spatial sample in a digital waveguide mesh (DWM) is represented by a $K$ port scattering junction, connected to its geometric neighbors over bidirectional unit-sample delay lines. In the typical case of an isotropic medium, $\mathbf{y}$ is a constant vector, while $\mathbf{A}$ and $\widetilde{\mathbf{A}}$ are identical and given by

$$
\mathbf{A}=\frac{2}{K} \mathbf{1 1}^{T}-\mathbf{I}
$$

We will refer to such a scattering matrix as the isotropic scattering matrix.

In the one-dimensional band-limited case, the DWM model provides the exact solution of the wave equation [34]. In the two [40] and three [41] dimensional cases, sound propagates in a DWM at slightly different speeds in different directions and different frequencies, causing a dispersion error [42], which can be controlled and reduced to some extent by means of careful design of the mesh topology or by using interpolated meshes and frequency warping methods [18], [43].

Accurate modeling with DWMs requires mesh topologies with a very fine resolution (e.g. $\approx 10^{7}$ junctions for a room of size $4 \times 6 \times 3 \mathrm{~m} \mathrm{[26]).} \mathrm{That} \mathrm{makes} \mathrm{the} \mathrm{computational} \mathrm{load} \mathrm{and}$ the amount of memory required prohibitively high for realtime operation, especially for large rooms. These drawbacks motivated the work of Karjalainen et al. reported in [26], which is reviewed in the next subsection.

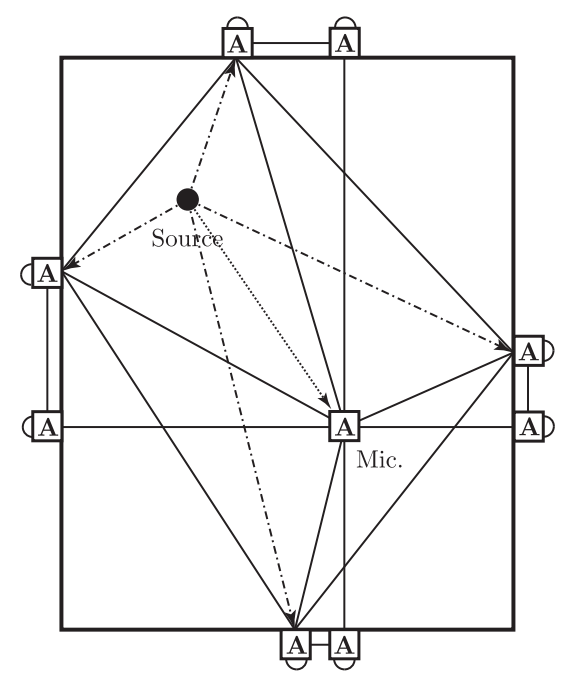

Fig. 3. Conceptual depiction of one of the DWN topologies proposed by Karjalainen et al. as seen by an observer above the simulated enclosure (in this case a 2D rectangular room) [26]. The solid black lines denote the bidirectional delay lines interconnecting scattering nodes. The scattering nodes are denoted by the $\mathbf{A}$ blocks, where $\mathbf{A}$ is the lossless scattering matrix. The dash-dotted lines denote the unidirectional absorptive delay lines. The dotted line denotes the line-of-sight (LOS) component. The solid arcs around junctions denote loaded self-connections implementing losses. Please note that while this figure represents the case of a $2 \mathrm{D}$ rectangular room, all the simulations in this paper use $3 \mathrm{D}$ rectangular rooms.

\section{Reduced Digital Waveguide Meshes}

In order to lower the computational complexity of DWM models, Karjalainen et al. considered coarse approximations of room response synthesis via sparse DWM structures [26]. One such structure is shown in Fig. 3. In this network, the sound source is connected via unidirectional absorbing delay lines to scattering junctions. These junctions are positioned at the locations where first-order reflections impinge on the walls. This ensures that delays of first-order reflections are rendered accurately. The junctions are connected via bidirectional delay lines with the microphone, which is also modeled as a scattering junction contributing to the energy circulation in the network. The line-of-sight component is modeled by a direct connection between the source and the microphone. Additional bidirectional delay lines parallel to the wall edges are included to better simulate room axial modes [44]. All the wall junctions are connected in a ring topology.

In order to model losses at the walls, it appears that a combination of junction loads and additional self-connections is used. However, implementation details are not given, and the authors state that the network required careful heuristic tuning [26]. For these reasons the results are difficult to replicate.

In the next section, we describe a structure which renders the direct path and first-order early reflections accurately both in time and amplitude, while producing progressively coarser approximations of higher order reflections and late reverberation. The proposed method has parameters that are inherited directly from room geometry and absorption properties of wall materials, and thus does not require tuning. In order to distinguish the proposed structure from the ones considered 


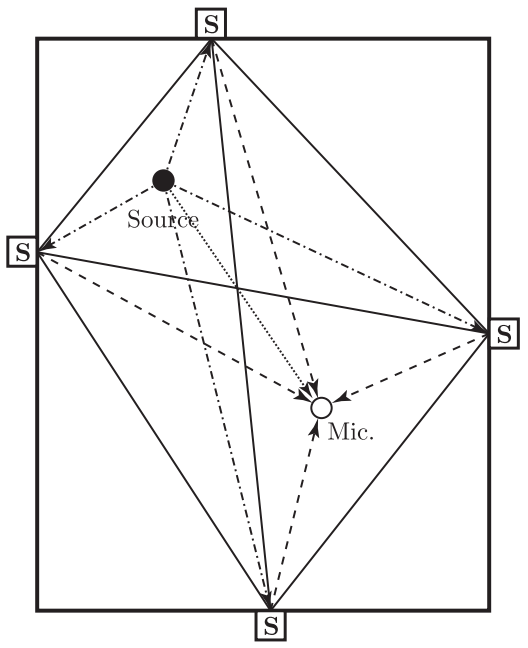

Fig. 4. Conceptual depiction of the SDN reverberator. The solid black lines denote bidirectional delay lines interconnecting the SDN wall nodes. The SDN wall nodes are denoted by the $\mathbf{S}$ blocks, where $\mathbf{S}$ is the lossy scattering matrix. The dash-dotted lines denote unidirectional absorptive delay lines connecting the source to the SDN nodes. The dashed lines denote unidirectional absorptive delay lines connecting the SDN nodes to the microphone. The dotted line denotes the direct-path component.

by Karjalainen et al. in [26], and because of the importance of the scattering operation, we refer to the proposed structures as scattering delay networks (SDNs).

\section{SCATtering Delay Networks}

The aim of the SDN structure is to simulate the acoustics of an enclosure using a minimal topology which would ensure that each significant reflection in the given space has a corresponding reflection in the synthesized response. This requires representing each significant reflective surface using one node in a fully connected mesh topology. The concept is illustrated in Fig. 4. For clarity, considerations in this paper will pertain to rectangular empty rooms, however all the presented concepts can be extended in a straightforward manner to arbitrary polyhedral spaces with additional reflective surfaces in their interior.

As shown in Fig. 4, the network consists of a fully connected DWN with one scattering node for each wall. This network is minimal in the sense that the removal of any of the nodes or paths would make it impossible to model a significant subset of reflections which would arise in the space.

The source is connected to the scattering nodes via unidirectional absorbing lines. As opposed to the reverberators proposed in [26], the microphone node is a passive element that does not participate in the energy recirculation, hence scattering nodes are connected to the microphone via unidirectional absorbing lines and no energy is injected from the microphone node back to the network.

Early reflections are known to strongly contribute to one's perception of the size and spaciousness of a room [45]. For this reason, nodes are positioned on the walls at locations of first-order reflections. Delays and attenuation of the lines connecting the nodes, source, and microphone are set so that

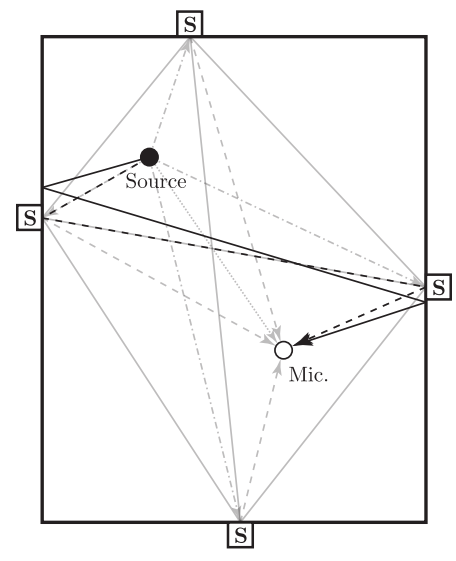

(a)

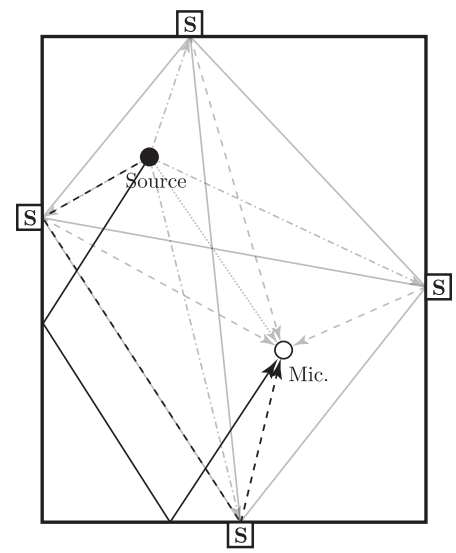

(c)

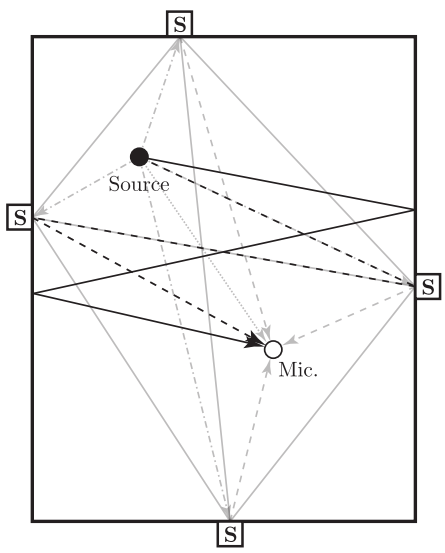

(b)

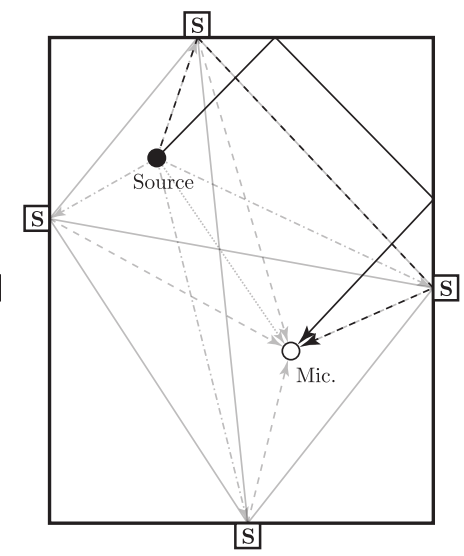

(d)
Fig. 5. Examples of approximations generated by SDN for four second-order reflections. The solid black line represents the actual path of the second-order reflection, while the dashed line is the corresponding path within the SDN.

first-order reflections are rendered accurately in their timing and energy.

Second-order reflections are approximated by corresponding paths within the network. This is illustrated in Fig. 5 It can be observed from the figure that the accuracy of second-order reflections depends on the particular reflection, but nonetheless the delays of the approximating paths are similar to the actual ones. As the reflection order increases, coarser approximations are made. Thus, the proposed network behaves equivalently to geometric-acoustic methods up to the first-order reflections, while providing a gracefully degrading approximation for higher-order ones.

Precise details of the SDN design are given below.

Scattering nodes: Each node is positioned on a wall of the modeled enclosure. The nodes are positioned at the locations where the first-order reflections impinge on the walls. These locations are straightforward to calculate for simple geometries, e.g. convex polyhedra. The nodes carry out a scattering operation on the inputs from the other $K$ nodes, $\mathbf{p}^{+}$, to obtain the outputs as $\mathbf{p}^{-}[n]=\mathbf{S p}^{+}[n]$, where $\mathbf{S}$ is the $K \times K$ (not necessarily lossless) scattering matrix. Rectangular rooms, which are used in the following, correspond to $K=5$. Other geometries where $K$ is a power or 2 are computationally 
convenient since it can be shown that they lead to a multiplierfree realization [11].

The scattering matrix $\mathbf{S}$ governs how energy circulates within the network. Since each node is associated with a wall, it describes how energy is exchanged between walls. Furthermore, when incident waves $\mathbf{p}^{+}$are scattered from the wall, a certain amount of energy is absorbed. A macroscopic quantity that describes material absorption sufficiently well for most synthesis applications is the random-incidence absorption coefficient [13]. This coefficient, specified by the ISO 354 standard, is known for a variety of materials [13].

The wall absorption effect can be expressed in the most general form as $\mathbf{p}^{-*} \mathbf{Y} \mathbf{p}^{-}=(1-\alpha) \mathbf{p}^{+*} \mathbf{Y} \mathbf{p}^{+}$, where $\alpha$ is the wall absorption coefficient, which is equivalent to

$$
\mathbf{S}^{*} \mathbf{Y} \mathbf{S}=(1-\alpha) \mathbf{Y}
$$

By expressing $\mathbf{S}$ as $\mathbf{S}=\beta \mathbf{A}$, where $\beta=\sqrt{1-\alpha}$, the relationship in $(10)$ becomes equivalent to $\mathbf{A}^{*} \mathbf{Y A}=\mathbf{Y}$, i.e. the scattering matrix $\mathbf{S}$ is the product of the wall reflection coefficient $\beta$ and a lossless scattering matrix $\mathbf{A}$. As in DWNs, if $\mathbf{A}$ is selected as given by (5) or (6), the propagating variables have a physical interpretation as the pressure or root-power waves in a network of acoustic tubes. Other non-physical choices of $\mathbf{A}$ are possible, as long as the lossless condition is satisfied; these are discussed in Section IV-C.

As will be shown in Section $\mathrm{V}-\mathrm{A}$, setting the scattering matrix as $\mathbf{S}=\beta \mathbf{A}$ results in a rate of energy decay that is consistent with the well-known Sabine and Eyring formulas as well as with the results of the IM. This can be attributed to the fact that both the average time between successive reflections (i.e. the mean free path) and the energy loss at the walls in the virtual SDN network are close to the ones observed in the corresponding physical room.

The absorption characteristic of most real materials is frequency-dependent. This can be modeled by using a more general scattering matrix $\mathbf{S}(z)=\mathbf{H}(z) \mathbf{A}$, where $\mathbf{H}(z)=$ $\operatorname{diag}\{H(z), \ldots, H(z)\}$, and $H(z)$ is a wall filter. The absorption coefficients in consecutive octave bands, for a range of materials, are reported in [13]. Standard filter design techniques can be used to fit the frequency response $H\left(e^{j \omega}\right)$ to these tabulated values. Minimum-phase IIR filters are particularly convenient in this context as they reduce computational load without significantly affecting the phase of simulated reflections [46].

As in conventional DWMs, the pressure at the SDN node is a function of the incoming wave variables, $p_{i}^{+}[n]$, from neighboring nodes and the pressure, $p_{S}[n]$, injected by the source. That is modeled according to (7), and it is illustrated in Fig. 6. Other details of source-to-node connections are discussed next.

Source-to-node connection: The input to the system is provided by a source node connected to SDN nodes via unidirectional absorbing delay lines (see Fig. 6).

The delay of the line connecting the source at $\mathbf{x}_{S}$ and the SDN node positioned at $\mathbf{x}_{k}$ is given by the corresponding propagation delay $D_{S, k}=\left\lfloor F_{s}\left\|\mathbf{x}_{S}-\mathbf{x}_{k}\right\| / c\right\rfloor$, where $c$ is the speed of sound and $F_{s}$ is the sampling frequency. The

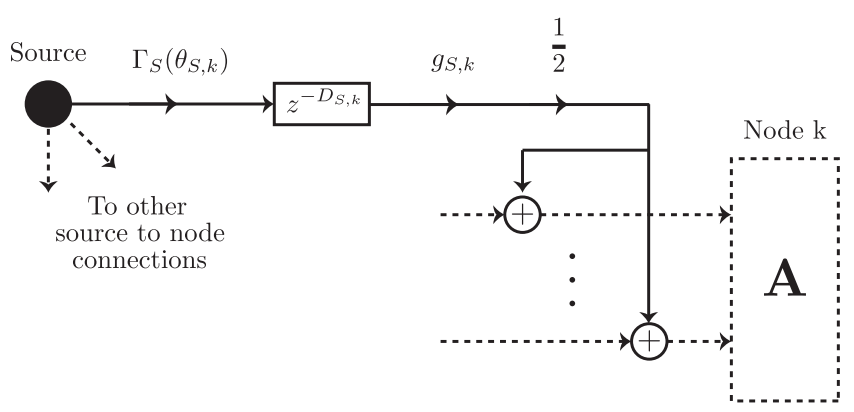

Fig. 6. Connection between the source node and an SDN node.

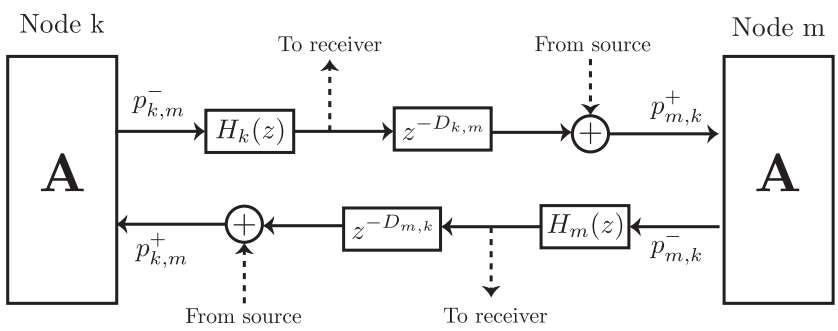

Fig. 7. Two interconnected SDN nodes.

attenuation due to spherical spreading $(1 / r$ law) is modeled as

$$
g_{S, k}=\frac{1}{\left\|\mathbf{x}_{S}-\mathbf{x}_{k}\right\|}
$$

Source directivity is another important simulation parameter in room acoustic synthesis. The sparse sampling of the simulated enclosure prohibits the simulation of source directivity in detail. However, a coarse approximation can be incorporated by weighting the outgoing signals by $\Gamma_{S}\left(\theta_{S, k}\right)$, where $\Gamma_{S}(\theta)$ is the source directivity, and $\theta_{S, k}$ is the angle between the source reference axis and the line connecting the source and $k$-th node. An alternative approach consists of using an average of the directivity pattern in some angular sector. It should be noted that it is possible to simulate frequency-dependent characteristics of source directivity using short, variable linear or minimum-phase filters. However, in order to keep the exposition in this article clear it is assumed that the directivity patterns are independent of frequency and can be modeled as simple gains.

Node-to-node connection: The connections between the SDN nodes consist of bidirectional delay lines modeling the propagation path delay as shown in Fig. 7 Additional low-pass filters can be inserted into the network at this point to model the frequency-dependent characteristic of air absorption, as proposed by Moorer in the context of FDNs [47].

The delays of the lines connecting the nodes are determined by their spatial coordinates. Thus, the delay of the line between a node at location $\mathbf{x}_{k}$ and a node at $\mathbf{x}_{m}$ is $D_{k, m}=\left\lfloor F_{s} \| \mathbf{x}_{k}-\right.$ $\left.\mathbf{x}_{m} \| / c\right\rfloor$.

Node-to-microphone connection: Each node is connected to the microphone node via a unidirectional absorbing delay line. The signal extracted from the junction, $p_{e}[n]$, is a linear combination of outgoing wave variables, $p_{e}[n]=\mathbf{w}^{T} \mathbf{p}^{-}[n]$, where $\mathbf{p}^{-}[n]$ is the wave vector after the wall filtering opera- 


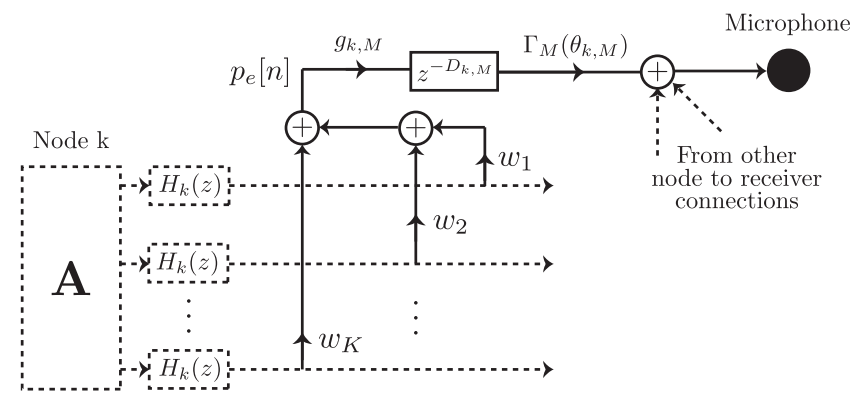

Fig. 8. Connection between an SDN node and the receiver/microphone node.

tion, as shown in Fig. 8

In the physical case where $\mathbf{A}$ is in the form (5) or (6), the outgoing signal to the microphone is taken as the node's pressure, as given by equations (4) and (7):

$$
p_{e}[n]=\frac{2}{\langle\mathbf{1}, \mathbf{y}\rangle} \mathbf{y}^{T} \mathbf{p}^{-}[n] \text {. }
$$

In the non-physical case, various choices are available for extracting a signal from the junction. The only condition that the weights $\mathbf{w}$ need to satisfy is that the cascade of pressure injection, scattering and extraction does not alter the amplitude of first-order reflections. Since the incoming wave vector $\mathbf{p}^{+}$ for a first-order reflection with amplitude $p_{S}$ is given by $\mathbf{p}^{+}=\left(\frac{p_{S}}{2} \mathbf{1}\right)$ (see Fig. 6 and since $\mathbf{p}^{-}=\mathbf{S} \mathbf{p}^{+}$, this condition can be written as $\mathbf{w}^{T} \mathbf{S}\left(\frac{p_{S}}{2} \mathbf{1}\right)=\beta p_{S}$ or, equivalently, as

$$
\mathbf{w}^{T} \mathbf{A} \mathbf{1}=2 .
$$

A possible choice for $\mathbf{w}$ is a constant vector, which is computationally convenient since it requires a single multiplication. In this case, the constraint (13) yields the unique solution $\mathbf{w}=\frac{2}{\mathbf{1}^{T} \mathbf{A} \mathbf{1}} \mathbf{1}$.

The delay from the $k$-th SDN node to the microphone node is $D_{k, M}=\left\lfloor F_{s}\left\|\mathbf{x}_{k}-\mathbf{x}_{M}\right\| / c\right\rfloor$. As with the source directivity, the microphone directivity is also modeled using a simple gain element $\Gamma_{M}\left(\theta_{k, M}\right)$, where $\Gamma_{M}(\theta)$ is the microphone directivity pattern and $\theta_{k, M}$ is the angle between the microphone acoustical axis and the $k$-th node. This approach ensures that the microphone is emulated correctly for the directions associated to the first-order reflections. As with the source-node connections, the microphone directivity can also be modeled using short, variable linear or minimum-phase filters. However, simple gain elements are preferred in this article for clarity of presentation. Similarly, as with the sourcenode connection, an alternative approach consists of using an average of the directivity pattern in some angular sector.

The attenuation coefficient is set as

$$
g_{k, M}=\frac{1}{1+\frac{\left\|\mathbf{x}_{k}-\mathbf{x}_{M}\right\|}{\left\|\mathbf{x}_{S}-\mathbf{x}_{k}\right\|}},
$$

such that, using (11),

$$
g_{S, k} \times g_{k, M}=\frac{1}{\left\|\mathbf{x}_{S}-\mathbf{x}_{k}\right\|+\left\|\mathbf{x}_{k}-\mathbf{x}_{M}\right\|},
$$

which yields the correct attenuation for the first-order reflection according to the $1 / r$ law. Notice that the above choice of $g_{k, M}$ and $g_{S, k}$ is not unique in satisfying the constraint 15 .
The attenuation can in fact be distributed differently between the source-to-node and node-to-microphone branches but with little impact on overall energy decay.

\section{Properties of SDNS}

\section{A. Transfer function}

The block diagram of an SDN system is shown in Fig. 9 In the figure,

$$
\begin{aligned}
\boldsymbol{\Gamma}_{S} & =\left[\Gamma_{S}\left(\theta_{S, 1}\right), \Gamma_{S}\left(\theta_{S, 2}\right), \ldots, \Gamma_{S}\left(\theta_{S, K+1}\right)\right]^{T}, \\
\mathbf{D}_{S}(z) & =\operatorname{diag}\left\{z^{-D_{S, 1}}, z^{-D_{S, 2}}, \ldots, z^{-D_{S, K+1}}\right\}, \\
\mathbf{G}_{S} & =\operatorname{diag}\left\{g_{S, 1}, g_{S, 2}, \ldots, g_{S, K+1}\right\},
\end{aligned}
$$

are the source directivity vector, the source delay matrix, and the source attenuation matrix, respectively. The corresponding quantities associated with the microphone $\boldsymbol{\Gamma}_{M}, \mathbf{D}_{M}(z)$ and $\mathbf{G}_{M}$ are defined as in (16), (17) and (18), by substituting $S$ with $M$. Further,

$$
\begin{aligned}
\mathbf{U} & =\operatorname{diag}\{\underbrace{\mathbf{1}, \ldots, \mathbf{1}}_{K+1}\}, \\
\mathbf{D}_{f}(z) & =\operatorname{diag}\left\{\left\{z^{-D_{1,2}}, \ldots, z^{-D_{K, K+1}}\right\},\right. \\
\overline{\mathbf{H}}(z) & =\operatorname{diag}\{\underbrace{H_{1}(z), \ldots, H_{1}(z)}_{K}, \ldots, H_{K+1}(z)\},
\end{aligned}
$$

are the block-diagonal matrix that distributes source signals to input wave variables, the inter-node delay matrix, and the wall absorption matrix, respectively. The block diagonal scattering matrix $\overline{\mathbf{A}}$ and the block diagonal weight matrix $\mathbf{W}$ are defined as

$$
\begin{aligned}
\overline{\mathbf{A}} & =\operatorname{diag}\left\{\mathbf{A}_{1}, \mathbf{A}_{2}, \ldots, \mathbf{A}_{K+1}\right\}, \\
\mathbf{W} & =\operatorname{diag}\left\{\mathbf{w}_{1}^{T}, \ldots, \mathbf{w}_{K+1}^{T}\right\},
\end{aligned}
$$

where $\mathbf{A}_{k}$ and $\mathbf{w}_{k}$ are the $i$-th node's scattering matrix and pressure extraction weights, respectively. While these variables can in general be different for each node, in all the simulations presented in this paper they are selected to be equal, $\mathbf{A}_{1}=$ $\cdots=\mathbf{A}_{K+1}$ and $\mathbf{w}_{1}=\cdots=\mathbf{w}_{K+1}$.

Finally, in Fig. 9. $g_{S, M}$ and $z^{-D_{S, M}}$ are the line-of-sight attenuation and delay, respectively, and $\mathbf{P}$ is a permutation matrix whose elements are determined based on the network topology. Due to the underlying node connectivity being bidirectional, this permutation matrix is symmetric. For the simplest case of a three-dimensional enclosure with a rectangular shape, the permutation matrix takes the form $\mathbf{P}=\delta_{i, f(j)}$, where $\delta_{i, j}$ is the Kronecker delta,

$$
f(i)=\left(\left(6 i-((i-1))_{N}-1\right)\right)_{N(N-1)}+1,
$$

and $((\cdot))_{N}$ is the modulo- $N$ operation. Inspection of Fig. 9 reveals that the system output can be expressed as

$$
Y(z)=\boldsymbol{\Gamma}_{M}^{T} \mathbf{D}_{M}(z) \mathbf{G}_{M} \mathbf{W} \mathbf{q}(z)+\bar{g} z^{-D_{S, M}} X(z)
$$

where $\bar{g}=g_{S, M} \Gamma_{S}\left(\theta_{S, M}\right) \Gamma_{M}\left(\theta_{M, S}\right)$, and $\mathbf{q}(z)$ is the state vector, given by

$\mathbf{q}(z)=\frac{1}{2}\left[\mathbf{I}-\overline{\mathbf{H}}(z) \overline{\mathbf{A}} \mathbf{P} \mathbf{D}_{f}(z)\right]^{-1} \overline{\mathbf{H}}(z) \overline{\mathbf{A}} \mathbf{U} \mathbf{G}_{S} \mathbf{D}_{S}(z) \boldsymbol{\Gamma}_{S} X(z)$. 


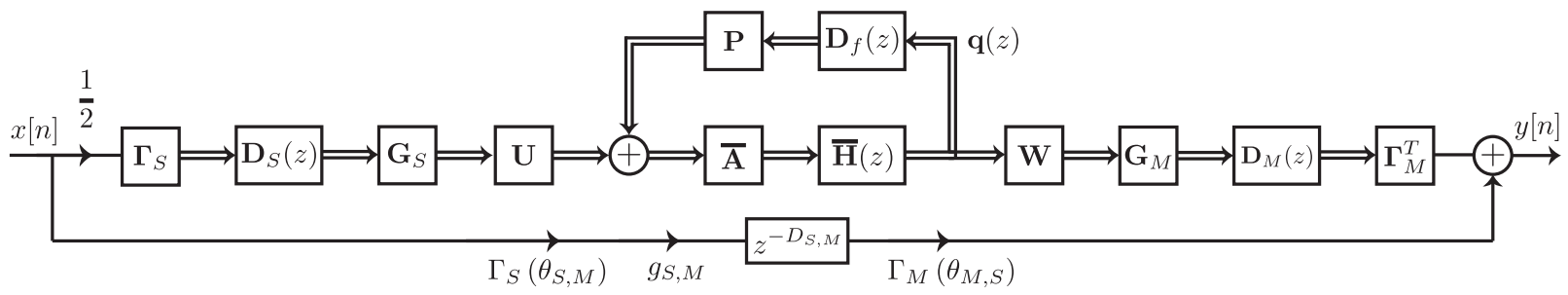

Fig. 9. Block diagram of the SDN reverberator.

The transfer function can therefore be expressed as

$H(z)=\frac{1}{K} \mathbf{k}_{M}^{T}(z)\left[\overline{\mathbf{A}}^{T} \overline{\mathbf{H}}^{-1}(z)-\mathbf{P} \mathbf{D}_{f}(z)\right]^{-1} \mathbf{k}_{S}(z)+\bar{g} z^{-D_{S, M}}$ where $\mathbf{k}_{M}^{T}(z)=\boldsymbol{\Gamma}_{M}^{T} \mathbf{D}_{M}(z) \mathbf{G}_{M} \mathbf{W}$ and $\mathbf{k}_{S}(z) \stackrel{(27)}{=}$ $\mathbf{U} \mathbf{G}_{S} \mathbf{D}_{S}(z) \boldsymbol{\Gamma}_{S}$.

It may be observed that, unlike FDN reverberators, relevant acoustical aspects, such as the direct path and reflection delays, are modeled explicitly, allowing complete control of source and microphone positions and their directivity patterns.

Expressing the system transfer function as given above allows for a complete demarcation of the directional properties and positions of the source and microphone, wall absorption characteristics and room shape and size. This is especially useful in keeping computational cost low for cases where only a single aspect, such as source orientation, changes.

\section{B. Stability}

The stability of the SDN follows from the fact that its recursive part, i.e. the backbone formed by the SDN nodes, is a fully connected DWN. The stability of lossless DWNs is, in turn, guaranteed by the fact that the network has a physical interpretation as a network of acoustic tubes [48], [10]. Indeed, the ideal physical system's total energy provides a Lyapunov function bounding the sum-of-squares in the numerical simulation (provided one uses rounding toward zero or error feedback in the computations) [48]. Furthermore, the network conserves its stability when losses due to wall absorption are included at the SDN nodes since the physical pressure (i.e. the sum of incoming and outgoing pressure waves) is then always reduced relative to the lossless case.

\section{Lossless Scattering Matrices}

In this section, we explore possible choices for lossless scattering matrices and discuss their implications. First we present a complete parametrization of real lossless matrices. The parameterization is an immediate corollary of the following theorem.

Theorem 1. A real square matrix $\mathbf{A}$ is diagonalizable if and only if it has the form

$$
\mathbf{A}=\mathbf{T}^{-1} \boldsymbol{\Lambda} \mathbf{T}
$$

where $\mathbf{T}$ is a real invertible matrix, and $\boldsymbol{\Lambda}$ is a block diagonal matrix consisting of $1 \times 1$ blocks which are real eigenvalues of $\mathbf{A}$, and $2 \times 2$ blocks of the form

$$
\left[\begin{array}{cc}
0 & -r_{i} \\
r_{i} & 2 r_{i} \cos \left(\theta_{i}\right)
\end{array}\right]
$$

where $r_{i} e^{j \theta_{i}}$ are complex eigenvalues of $\mathbf{A}$ which appear in pairs with their conjugates.

The theorem is proved in the appendix.

Corollary 2. A real square matrix is lossless if and only if it has the form given by Theorem 1 with all eigenvalues on the unit circle.

The corollary follows from the fact that a square matrix is lossless if and only if it is diagonalizable and all its eigenvalues are on the unit circle [10].

Within the space of lossless matrices, a large degree of leeway is left for pursuing various physical or perceptual criteria. This can be achieved, for instance, by finding a lossless matrix $\mathbf{A}$ which minimizes the distance from a real matrix $\mathbf{D}$ that reflects some sought-after physical or perceptual properties. The design then amounts to constrained optimization:

$$
\min _{\mathbf{A}}\|\mathbf{A}-\mathbf{D}\|_{F}^{2}
$$

where $\|\cdot\|_{F}$ denotes the Frobenius norm, under the constraint that $\mathbf{A}$ has the form given in Corollary 2. This most general case may, however, involve optimization over an excessive number of parameters. If we restrict the optimization domain to orthogonal matrices, solutions can be found without the need for numerical procedures. In particular, the following Theorem result holds:

Theorem 3. The solution to the following optimisation problem

$$
\underset{\mathbf{A}}{\operatorname{argmin}}\|\mathbf{A}-\mathbf{D}\|_{F}^{2}, \mathbf{A}^{T} \mathbf{A}=\mathbf{I}
$$

is given by $\mathbf{A}=\mathbf{U V}^{T}$, where $\mathbf{U}$ and $\mathbf{V}$ are respectively the matrices of left and right singular vectors of $\mathbf{D}$.

A proof of this result can be found in [49].

Orthogonal matrices which are also circulant have the interpretation of performing all-pass circulant convolution of the incoming variables, which as discussed below, reduces computational complexity of the scattering operator. Furthermore, if a certain distribution of (unit-norm) eigenvalues is sought, the associated circulant matrix can be found by means of a single inverse fast Fourier transform (FFT) [10]. An indepth study of such scattering matrices in the context of DWN reverberators has been presented in [10]. 
Householder reflection matrices, given by $\mathbf{A}=2 \mathbf{v v}^{T}$ I, $\|\mathbf{v}\|=1$, are the subclass of orthogonal scattering matrices commonly used in the context of DWN reverberators. As discussed in Section II-B they enable a physical interpretation of the propagating variables as normalized pressure waves in a network of acoustic tubes. The optimization problem that minimizes the distance from a targeted scattering matrix in this case can be expressed as

$$
\underset{\mathbf{v}}{\operatorname{argmin}}\left\|\left(2 \mathbf{v} \mathbf{v}^{T}-\mathbf{I}\right)-\mathbf{D}\right\|_{F}^{2},\|\mathbf{v}\|=1,
$$

the solution of which is given by the following theorem.

Theorem 4. The solution to the optimization problem in 317 is the singular vector corresponding to the largest singular value of matrix $\mathbf{D}+\mathbf{D}^{T}$.

The theorem is proved in the appendix.

The isotropic scattering matrix, i.e. the particular case of a Householder reflection obtained for $\mathbf{v}=1 / \sqrt{K}$, can be physically interpreted as the scattering matrix which takes a reflection from one node and distributes its energy equally among all other nodes. This is the only orthogonal matrix which has this property, as stated by the following theorem.

Theorem 5. If $\mathbf{A}$ is an orthogonal matrix which scatters the energy from each incoming direction uniformly among all other directions, then it must have the form $\mathbf{A}= \pm \frac{2}{K} \mathbf{1 1}^{T}-\mathbf{I}$.

The theorem is proved in the appendix. The isotropic matrix thus satisfies some optimality criteria and, as discussed below, allows for fast implementation. We will see in Section V-B that its special structure leads to a fast build up of echo density which is a perceptually desirable quality [29].

These different choices of scattering matrices have their implications on computational complexity. The computational complexity of the matrix-vector multiplication using general lossless and orthogonal matrices is $O\left[K^{2}\right]$ operations. Circulant lossless matrices require only $O[K \log (K)]$ operations [10]. The computational complexity associated with matrices which have the form of Householder reflections is further reduced to $O[K]$. Among general Householder reflections, which require $2 K-1$ additions and $2 K$ multiplications, the isotropic scattering matrix only requires $2 K-1$ additions and 1 multiplication. Among lossless matrices, the ones that require the least operations are permutation matrices. However, we will see in Section $\mathrm{V}-\mathrm{B}$ that permutation matrices lead to an insufficient echo density.

\section{Interactivity and multichannel auralization}

Interactive operation of the SDN reverberator is accomplished by updating the model to reflect changes in the positions and rotations of the source and microphone. This requires readjusting the positions of the wall nodes, and updating the delay line lengths and gains accordingly. It was observed in informal listening tests that updating the delay line lengths did not cause audible artifacts as long as microphone and source speeds are within reasonable limits.

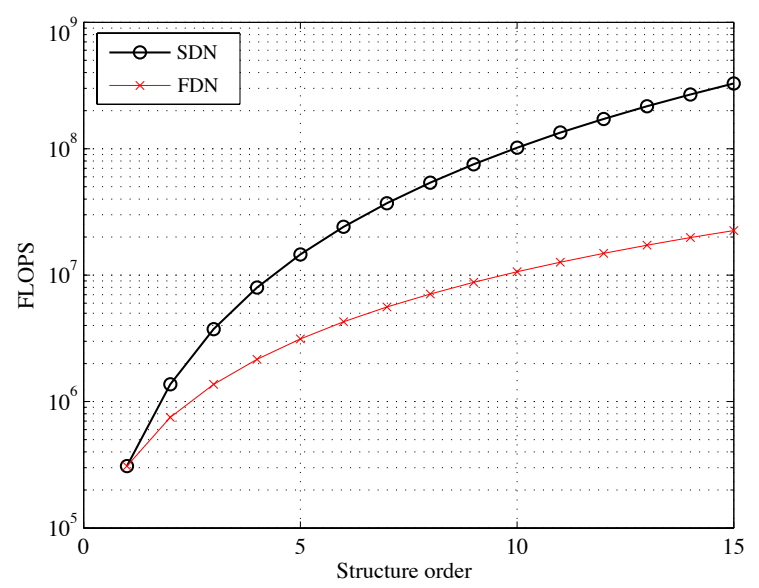

Fig. 10. Comparison of computational complexity for SDN and FDN as a function of the structure order, i.e. the size of the feedback matrix for FDN and the number of neighboring nodes $K$ for SDN (notice that the case of rectangular rooms corresponds to $K=5$ ). The sampling frequency is $F_{s}=44100 \mathrm{~Hz}$.

The proposed model allows approximate emulation of coincident and non-coincident recording formats. Coincident formats (e.g. Ambisonics [21], higher-order ambisonics (HOA) [50], vector-base amplitude panning (VBAP) [51]) can be easily employed by adjusting the microphone gains $\Gamma_{M, k}(\theta)$ appropriately.

Non-coincident formats (e.g. [52], [53]) can be emulated by considering a separate SDN for each microphone. This results in a higher inter-channel decorrelation, which is what would actually occur in real recordings. However, the overall computational load also increases. If simulation speed is critical, an alternative approach would be to share the same set of wall nodes among all the SDNs, while creating dedicated node-to-microphone connection lines for each microphone.

\section{E. Computational load}

This section presents an analysis of the computational complexity of the proposed model in comparison to the two conceptually closest technologies, FDN and the IM. We use the number of floating point operations per second as an approximate indicator of the overall computational complexity. Furthermore, to simplify calculations we make the assumption that additions and multiplications carry the same cost. This approximation is motivated by the progressive convergence of computation time of various operations in modern mathematical processing units.

The number of floating point operations per second (FLOPS) performed by an SDN can be calculated as $F_{s}\left[2 K^{3}+(P+2) K^{2}+K+1\right]$, where $P$ is the number of operations required by each wall filter for each sample. Consider now an FDN with a $Q \times Q$ feedback matrix. From inspection of Fig. 1 the computational complexity can be shown to be $F_{s}\left[2 Q^{2}+(P+3) Q+1\right]$ FLOPS. Fig. 10 shows a comparison of the computational complexity of SDNs and FDNs. In this figure, the $\mathrm{x}$-axis denotes the structure order, which we define as the number of neighboring nodes $K$ for SDN and the size of the feedback matrix $Q$ for FDN. The 
sampling frequency is set to $F_{s}=44100 \mathrm{~Hz}$, and the filtering step consists of a simple frequency independent gain $(P=1)$ for both SDN and FDN. It may be observed that for the typical case of a 3D rectangular room $(K=5), \mathrm{SDN}$ has around the same computational complexity of an FDN with a $12 \times 12$ feedback matrix. More specifically, SDN for $K=5$ has a complexity of 14.6 MFLOPS, while FDN for $Q=12$ has a complexity of 14.9 MFLOPS. Notice that the computational complexity of both SDN and FDN can be reduced significantly by using efficient lossless matrices of the type discussed in Section IV-C e.g. Householder and circulant lossless matrices.

Consider now the computational cost of the IM method for rectangular rooms in its original time-domain implementation [15]. In the IM, each image source requires 10 floatingpoint operation $\leqslant$ to calculate the time index and $1 / r$ attenuation of the image, and 15 floating-point operations to calculate its attenuation due to wall absorption. This amounts to 25 floating-point operations in total for each image source. The number of image sources contained in an impulse response of length $T_{60}$ seconds is approximately equal to the number of room cuboids that fit in a sphere with radius $c T_{60}$ meters. This gives a computational complexity of around

$$
25\left\lceil\frac{\frac{4}{3} \pi\left(T_{60} c\right)^{3}}{L_{x} L_{y} L_{z}}\right\rceil \text { FLOPS }
$$

where $L_{x}, L_{y}$ and $L_{z}$ are the room dimensions. Here, we are implicitly assuming that the entire RIR is calculated using the IM. While this ensures a fair comparison in terms of the other properties assessed in the next section, it should be noted that, in order reduce the complexity, most auralization methods use the IM only to simulate the early reflections. This is especially so in case of non-rectangular rooms, which require a considerably larger amount of memory and computational power than equation (32) [16]. The rest of the RIR is usually generated using lightweight statistical methods, e.g. random noise with appropriate energy decay [54].

Once the RIR has been generated, it has to be convolved with the input signal to obtain the reverberant signal. In the case of real-time applications, this can be done efficiently using the overlap-add method [55]. The method calculates 2 FFTs of length $N, N$ complex multiplications, 1 inverse FFT, and $\left\lceil T_{60} F_{s}\right\rceil$ real additions for each time frame. In order for the circular convolution to be equal to the linear convolution, $N$ must satisfy $N \geq\left\lceil F_{s} / F_{r}\right\rceil+\left\lceil T_{60} F_{s}\right\rceil-1$, where $F_{r}$ is the frame refresh rate. In the best-case scenario where $N$ is a power of 2, the asymptotic cost of each FFT is $6 N \log _{2} N$ [55]. Furthermore, each complex multiplication requires 4 real multiplications and 2 additions. Overall, the computational complexity of the overlap-add method is $F_{r}\left(18 N \log _{2} N+6 N+\left\lceil T_{60} F_{s}\right\rceil-1\right)$ FLOPS. In the static case, the FFT of the impulse response can be precomputed, and the cost reduces to $F_{r}\left(12 N \log _{2} N+6 N+\left\lceil T_{60} F_{s}\right\rceil-1\right)$ FLOPS.

Fig. 11 compares the cost of SDN with the static and dynamic IM. In the static case, both microphone and source are not moving. In the dynamic case, on the other hand,

\footnotetext{
${ }^{1}$ Here we assume that exponentiations and square roots count as a single floating-point operation.
}

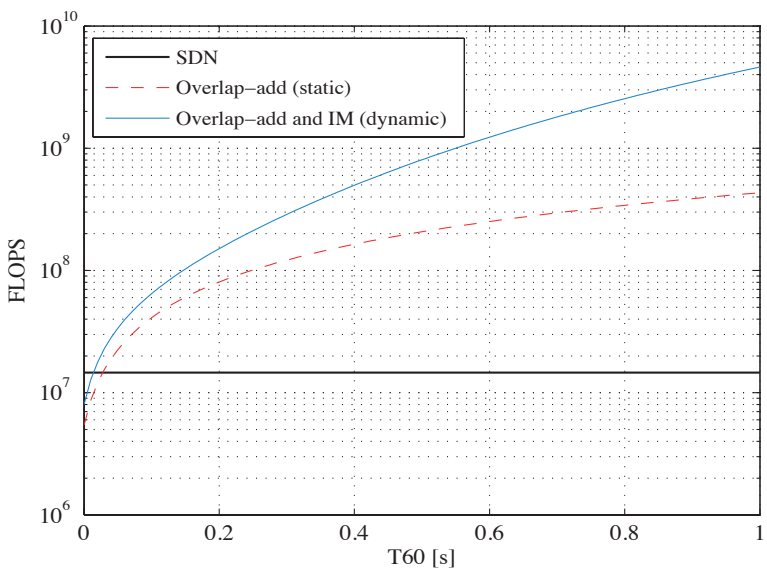

Fig. 11. Computational complexity of SDN in comparison to overlap-add convolution in both static and dynamic modes as a function of reverberation time. The static case represents the cost of the overlap-add convolution with a fixed, precomputed RIR. The dynamic case also includes the cost of calculating a new RIR and its FFT for each time frame. The sampling frequency is $F_{s}=44100 \mathrm{~Hz}$.

microphone and/or source are moving, and the IM is run for each frame. The refresh rate is chosen such that the buffering delay is shorter than the maximum latency of a half-frame delay between the video and audio, as recommended by the ITU Recommendation BR.265-9 [56]. For a video running at 25 frames per second, this criterion gives a refresh rate of $F_{r}=50 \mathrm{~Hz}$. The room size is the same as used in Allen and Berkley's paper of $10 \times 15 \times 12.5$ feet [15]. It may be observed that for typical medium-sized rooms, SDN is from about 10 to 100 times faster than dynamic IM. SDN is also significantly faster than (overlap-add) convolution alone.

While we compare the computational complexity of the proposed algorithm with the standard overlap-add convolution, we also acknowledge that more efficient convolution methods have recently been proposed, e.g. [57]-[59]. However, the comparison of the proposed algorithm with these new methods is outside the scope of this article.

\section{F. Memory requirement}

The required memory is determined by the number of taps of the delay lines. An upper bound for memory requirement $Q$ can be easily found by observing that the length of each delay line is smaller than or equal to the distance between the two farthest points of the simulated space, giving:

$$
Q \leq(N(N-1)+2 N+1) \frac{q F_{s}}{c} R \text { bits },
$$

where $q$ is the number of bits per sample, and $R$ is the maximum distance between any two points in the simulated space. The value of $R$ in the case of a rectangular room is $R=\sqrt{L_{x}^{2}+L_{y}^{2}+L_{z}^{2}}$. For the more general case, $R$ is the diameter of the bounding sphere of the room shape.

Observe in 33 that $Q$ scales linearly with the room size. For a cubic room with a $5 \mathrm{~m}$ edge, $F_{s}=40 \mathrm{kHz}$, and $q=$ 32 bytes per sample, the memory requirement is less than $170 \mathrm{kB}$, which is negligible for virtually every state-of-the-art platform. 


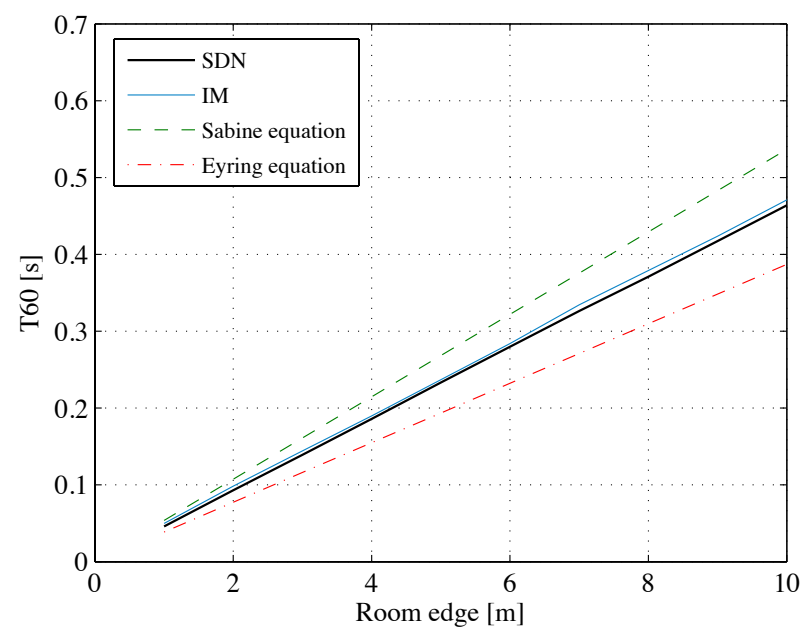

Fig. 12. Values of reverberation time $T_{60}$ as a function of the edge of a cubic room. The wall absorption is $\alpha=0.5$, and both microphone and source are positioned in the volumetric center of the room.

\section{Assessment}

This section presents the results of assessments of SDNs in terms of perceptually-based objective criteria.

As discussed in previous sections, first-order reflections are rendered correcty both in timing and amplitude by construction. Another cue important for the perception of room volume and materials is the reverberation time [45]. Section $\mathrm{V}$-A presents an evaluation of the reverberation time both in frequency-independent and frequency-dependent cases. Section $\mathrm{V}-\mathrm{B}$ focuses on the time evolution of echo density, which is related to the perceived time-domain texture of reverberation [29].

Here, the IM is used as a reference since it is the closest method among physical room models. More specifically, we use a C++ version of Allen and Berkley's original time-domain implementation [15].

\section{A. Reverberation time}

The parameter most commonly used to quantify the length of reverberation is $T_{60}$, which is defined as the time it takes for the room response to decay to $60 \mathrm{~dB}$ below its starting level. In this section, the $T_{60}$ of the SDN network is compared with two well-known empirical formulas proposed by Sabine [60] and Eyring [61]:

$$
\begin{aligned}
T_{60, \text { Sab }} & =\frac{0.161 \mathrm{~V}}{\sum_{i} A_{i} \alpha_{i}}, \\
T_{60, \text { Eyr }} & =-\frac{0.161 \mathrm{~V}}{\left(\sum_{i} A_{i}\right) \log _{10}\left(1-\sum_{i} A_{i} \alpha_{i} / \sum_{i} A_{i}\right)},
\end{aligned}
$$

where $V$ is the room volume, $A_{i}$ and $\alpha_{i}$ are the area and absorption coefficient of the $i$-th wall, respectively.

1) Frequency-independent wall absorption: Cubic rooms with different volumes and uniform frequency-independent absorption are simulated. In order to maintain the experimental conditions across different room sizes, both the source and the microphone are placed at the volumetric center of the

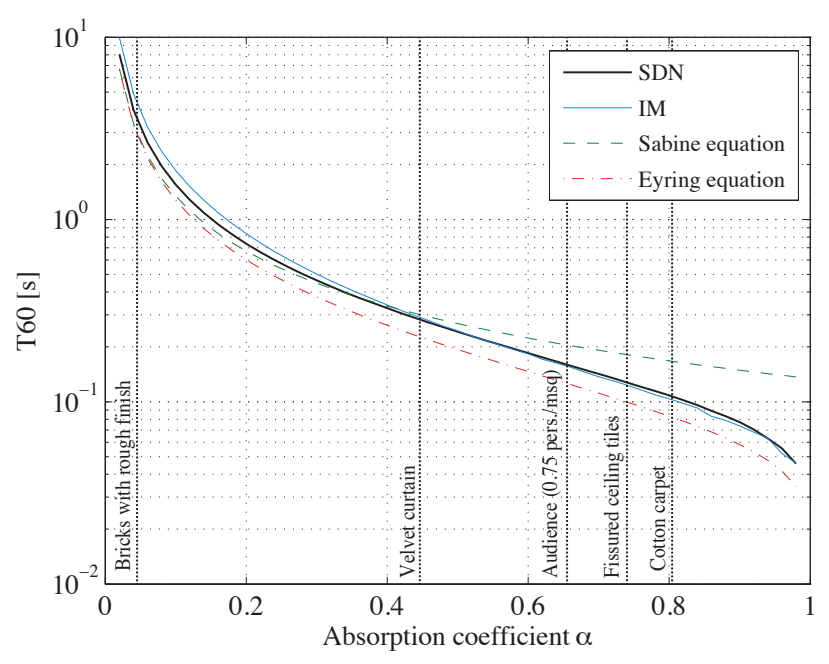

Fig. 13. Reverberation time values as a function of the absorption coefficient $\alpha$ for SDN, IM, and Sabine and Eyring predictions. The simulated enclosure is a cube with $5 \mathrm{~m}$ edge. $T_{60}$ values are averaged across 10 source and microphone random positions. The $1 \mathrm{kHz}$ absorption coefficient of various materials as measured by Vorländer in [13] are reported at the bottom of the plot as reference.

room. Furthermore, the line-of-sight component was removed, as suggested in the ISO 3382 standard [62] for measuring the reverberation time in small enclosures. In Fig. 12 the reverberation time is shown as a function of the edge length for a room absorption coefficient $\alpha=0.5$. It may be observed that the SDN generates room impulse-responses which have reverberation times that increase linearly with the edge length. This is due to the larger average distance between the nodes, which in turn increases the mean free path of the structure.

The $T_{60}$ values corresponding to the SDN reverberator are between the predictions given by Sabine and Eyring's formulas and are nearly identical to the ones produced by the IM. The latter result may seem surprising if one considers that the SDN, as opposed to the IM, does not include attenuation due to spherical spreading (except for the initial first-order reflections and microphone taps). This apparent inconsistency is resolved intuitively by observing that spherical spreading is a lossless phenomenon: In the IM, the quadratic energy decrease $\left(1 / r^{2}\right)$ is compensated by the quadratic increase of the number of contributing image sources over time, and similarly to that, in DWN and SDN, "plane waves" are scattered losslessly at each node, thus conserving the energy.

In Fig. 13 the reverberation time is shown as a function of the absorption coefficient $\alpha$. The enclosure was taken as a cubic room with a $5 \mathrm{~m}$ edge, and results were averaged across 10 pairs of source-microphone positions. The coordinates were taken from a uniform random distribution and satisfied both requirements set in [62]: The microphone was at least $1 \mathrm{~m}$ away from the nearest wall, and the distance between the source and microphone was at least

$$
d_{\text {min }}=2 \sqrt{\frac{V}{c T_{e s t}}}
$$

where $T_{e s t}$ is a coarse estimation of the reverberation time. In these simulations, $T_{\text {est }}$ was set using Sabine's formula. 


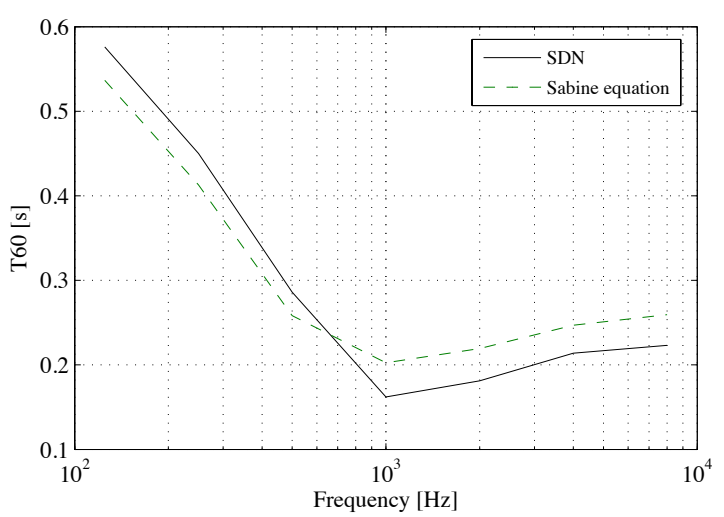

Fig. 14. Comparison of reverberation time in different octave bands for SDN and Sabine's formula prediction.

It may be observed that for absorption coefficients higher than around $\alpha=0.4, \mathrm{SDN}$ and the IM are nearly identical and are both between Sabine and Eyring's formulas. For high absorption coefficients, both SDN and the IM approach Eyring's formula, which is known to give more accurate predictions in that region [61]. For low absorption coefficients, SDN and the IM produce reverberation times longer than Sabine and Eyring's formulas, with SDN being closer to both.

2) Frequency-dependent wall absorption: Fig. 14 shows the result of a simulation where all walls mimic the frequencydependent absorption of cotton carpet. The filters $H_{i}(z)$ are all set to be equal to a filter $H(z)$ which was implemented as a minimum-phase IIR filter with coefficients optimized by a damped Gauss-Newton method to fit the absorption coefficients reported by Vorländer in [13]. This procedure gave

$$
H(z)=\frac{0.6876-1.9207 z^{-1}+1.7899 z^{-2}-0.5567 z^{-3}}{1-2.7618 z^{-1}+2.5368 z^{-2}-0.7749 z^{-3}} .
$$

The source and microphone were positioned on the diagonal of a cubic room with $5 \mathrm{~m}$ edge. More specifically, they were positioned on the diagonal at a distance of $d_{\min }=2.96 \mathrm{~m}$ from the center, as specified using 36.

In Fig. 14 the wall filter response is plotted together with the corresponding Sabine predictions in (34), which for the given room becomes

$$
T_{60, S a b}=\frac{0.161 V}{\sum_{i} A_{i} \alpha_{i}(\omega)}=\frac{0.161 \cdot 5}{6 \alpha(\omega)}=\frac{0.161 \cdot 5}{6\left(1-\left|H\left(e^{j \omega}\right)\right|^{2}\right)} .
$$

The simulated RIR was fed into an octave-band filter bank, and $T_{60}$ values were calculated for each octave-band. As shown in Fig. 14 these measured $T_{60}$ are very close to Sabine's formula prediction, thus confirming that the proposed model allows controlling the absorption behavior of wall materials explicitly.

Note that if explicit control of the reverberation time is sought, the prediction functions (34) or 35 can be inverted to obtain needed $\alpha(\omega)$. To this end, Eyring's formula 35 is preferable, since (34) may yield non-physical values for the absorption coefficient (i.e. $\alpha>1$ ) of some acoustically "dead" rooms [61].

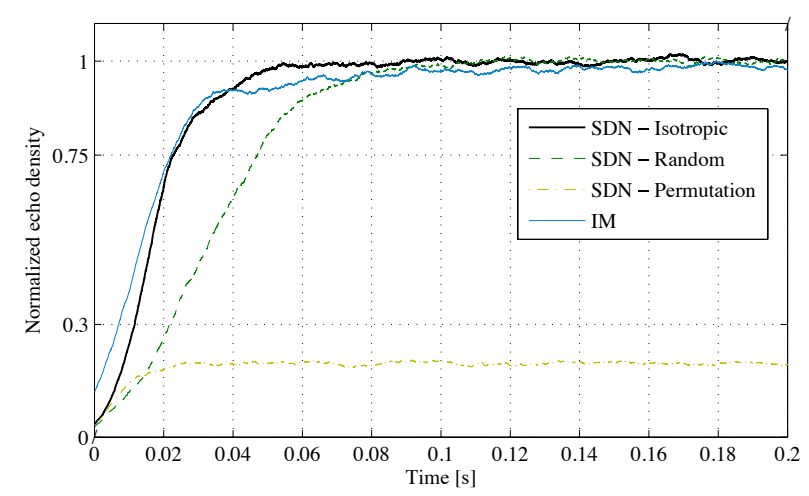

Fig. 15. Time evolution of the normalized echo density for the IM and SDN with various scattering matrices $\mathbf{S}$.

\section{B. Echo density}

The time evolution of echo density is commonly thought to influence the perceived time-domain texture of reverberation [29]. In an effort to quantify this perceptual attribute, Abel and Huang defined the normalized echo density (NED), which was found to have a very strong correlation with results of listening tests [29]. The NED is defined as the percentage of samples of the room impulse response lying more than a standard deviation away from the mean in a given time window compared to that expected for Gaussian noise. A NED equal to 1 means that, within the considered window, the number of samples lying more than one standard deviation away from the mean is equal to the one observed with Gaussian noise.

Fig. 15 shows the time evolution of the NED obtained with the IM and with the proposed model using three different scattering matrices. The scattering matrices are (a) the isotropic matrix, (b) a random orthogonal matrix, and (c) a random permutation matrix. The random orthogonal matrix was obtained by setting the angles of a Givens-rotation parametrization of orthogonal matrices [63] at random. The simulated enclosure was a rectangular room with dimensions $3.2 \times 4.0 \times 2.7 \mathrm{~m}$, and results were averaged across 50 random pairs of source and microphone positions. The wall gains were set to $\beta=-\sqrt{0.9}$ (wall absorption of $\alpha=0.1$ ), with the negative sign being chosen in order to obtain a zero-mean reverberation tail with the IM.

Fig. 15 shows that the build-up of echo density of SDN is very close to that of the IM when the isotropic scattering matrix is employed. In particular, the NED values of 0.3 and 0.75 , which were previously identified as breakpoints dividing three perceptually distinct groups [29], are reached at around the same delays by the two methods. Notice how the permutation matrix fails to reach a Gaussian-like reverberation. The random orthogonal matrix, on the other hand, does reach a Gaussian-like reverberation, but it takes longer to achieve the desired reverberation quality characterized by the 0.75 breakpoint.

\section{Mode density}

The mode density, i.e. the average number of resonant frequencies per $\mathrm{Hz}$, is another important perceptual property 
in artificial reverberation [6]. In order to achieve a naturalsounding reverberation, the mode density should be sufficiently high, such that no single resonance stands out causing metallic-sounding artifacts. A threshold for the minimum mode density that is commonly used in this context is [6]:

$$
d_{\text {min }}=\frac{T_{60}}{6.7}
$$

which is due to an early work of Schroeder [64].

The mode density in SDN can be calculated using considerations similar to those used for FDNs [6], [34]. Assuming that the wall filters are simple gains, i.e. $\overrightarrow{\mathbf{H}}(z)=\beta \mathbf{I}$, and applying the inversion lemma to the transfer function (27), the poles of the system can be calculated as the solutions of

$$
\operatorname{det}\left(\mathbf{D}_{f}\left(z^{-1}\right)-\beta \overline{\mathbf{A}} \mathbf{P}\right)=0 .
$$

Using Leibniz's formula for determinants it is easy to see that the order of the polynomial in 39 ) is equal to the summation of all the delay-line lengths in the SDN backbone, i.e. $\sum_{i} \sum_{j \neq i} D_{i, j}$. Using the fundamental theorem of algebra, and assuming that the poles are uniformly distributed [34], the mode density of the SDN network can be calculated as

$$
d_{f}=\frac{1}{F_{s}} \sum_{i} \sum_{j \neq i} D_{i, j} .
$$

The SDN structure satisfies $d_{f}>d_{\min }$ under most conditions of practical interest. This can be easily shown analytically in the case where source and microphone are close to the volumetric center of a cubic room with edge $L$. In this case, the length of the delay lines is approximately $L \frac{F_{s}}{c}$ for the six lines connecting opposite walls and $\frac{\sqrt{2}}{2} L \frac{F_{s}}{c}$ for the remaining twenty four lines. The mode density is thus

$$
d_{f}=\left(6+24 \frac{\sqrt{2}}{2}\right) \frac{L}{c} \approx 23 \frac{L}{c} .
$$

The condition $d_{f}>d_{\min }$ is then satisfied whenever

$$
L>\frac{c}{6.7 \times 23} T_{60} \approx 2.22 T_{60} .
$$

Since practical $T_{60}$ values for reverberation are on the order of a second, it follows that SDNs has a sufficient mode density as long as $L>2.22 \mathrm{~m}$ (i.e. volume larger than around $11 \mathrm{~m}^{3}$ ), which covers most cases of practical interest.

By replacing $T_{60}$ in (42) with Sabine's approximation (34), it can also be seen that the condition $d_{f}>d_{\min }$ is satisfied whenever the absorption coefficient is larger than $\alpha>0.06$, or, equivalently, $\beta<0.97$.

In order to assess the mode density in cases more general than the cubic one, Monte Carlo simulations were run using rectangular rooms with randomly selected parameters. More specifically, the three room dimensions were each drawn from a uniform distribution between 2 and 10 meters. The absorption coefficient was common to all walls and was drawn from a uniform distribution between 0 and 1 . The microphone and the source were placed in positions chosen at random within the room boundaries. Out of 1000 simulations, SDN provided a sufficient mode density in the $94.7 \%$ of cases. Among the cases that did not provide sufficient echo density, the largest absorption coefficient was $\alpha=0.066$, which is largely in agreement with the result obtained above for cubic rooms.

\section{CONClusions And Future Work}

This paper presented a scalable and interactive artificial reverberator termed scattering delay network (SDN). The room is modeled by scattering nodes interconnected by bidirectional delay lines. These scattering nodes are positioned at the points where first-order reflections originate. In this way, the firstorder reflections are simulated correctly, while a rich but less accurate reverberation tail is obtained. It was shown that, according to various objective measures of perceptual features, SDN achieves a reverberation quality similar to that of the IM while having a computational load one to two orders of magnitude lower.

The interested reader can listen to SDN-generated audio samples that are made available as supplementary downloadable material at http://ieeexplore.ieee.org and at [65].

Several directions for future research can be envisioned on the basis of the work presented in this paper. The design of the backbone network in Fig. 4 has a simple geometrical interpretation. However, the lengths of the delay lines in the backbone network can be designed using different approaches. It is believed, in fact, that, as long as the network has a meanfree-path similar to the one of the corresponding physical space, the SDN will conserve its appealing properties observed in Sec. $\mathrm{V}$ The delay-line lengths could be designed, for instance, to minimize the average timing error of higher-order reflections or to achieve a better fit with the modal frequencies of the physical space. The number of SDN nodes could also be increased. Indeed, while using a single SDN node for each wall is the minimum to ensure that all higher-order reflections are modeled, a larger number of nodes could be used, for instance, to further increase the modal density or to emulate higher-order reflections exactly. This would, of course, come at the expense of an increased computational complexity.

\section{APPENDIX}

Towards proving Theorem 1, we first establish the following lemma.

Lemma 6. Two diagonalizable matrices have the same eigenvalues if and only if they are similar.

Proof of Lemma 65 The sufficiency is a well know result on similar matrices [66]. To prove the necessity, let us consider two diagonalizable matrices $\mathbf{A}$ and $\mathbf{B}$ which have the same eigenvalues. Since $\mathbf{A}$ is diagonalizable, it follows that $\mathbf{V}_{A}^{-1} \mathbf{A} \mathbf{V}_{A}=\boldsymbol{\Lambda}$, where $\boldsymbol{\Lambda}$ is a diagonal matrix and $\mathbf{V}_{A}$ is an invertible matrix. Hence, the following equality holds: $\mathbf{A} \mathbf{V}_{A}=\mathbf{V}_{A} \boldsymbol{\Lambda}$, which implies that $\boldsymbol{\Lambda}$ has eigenvalues of $\mathbf{A}$ on its diagonal. Since $\mathbf{B}$ is also diagonalizable and has the same eigenvalues as $\mathbf{A}$, it satisfies $\mathbf{V}_{B}^{-1} \mathbf{B} \mathbf{V}_{B}=\boldsymbol{\Lambda}$. Thus, $\mathbf{V}_{A}^{-1} \mathbf{A} \mathbf{V}_{A}=\boldsymbol{\Lambda}=\mathbf{V}_{B}^{-1} \mathbf{B} \mathbf{V}_{B}$, which further implies that $\mathbf{A}=\left(\mathbf{V}_{B} \mathbf{V}_{A}^{-1}\right)^{-1} \mathbf{B}\left(\mathbf{V}_{B} \mathbf{V}_{A}^{-1}\right)$, i.e. the two matrices are similar.

Using Lemma 6 we can now prove Theorem 1: 
Proof of Theorem [1. First, observe that $\boldsymbol{\Lambda}$ is block diagonal, and that eigenvalues of each block are mutually distinct. Hence, each block of $\boldsymbol{\Lambda}$ is diagonalizable, and therefore $\Lambda$ is itself diagonalizable [66] (over the field of complex numbers, $\mathbb{C}$ ). Thus, $\boldsymbol{\Lambda}$ has the same eigenvalues as $\mathbf{A}$ and is diagonalizable. Since $\boldsymbol{\Lambda}$ and $\mathbf{A}$ are both diagonalizable, and have the same eigenvalues, according to Lemma 6 they must be similar over $\mathbb{C}$. Moreover, since both $\boldsymbol{\Lambda}$ and $\mathbf{A}$ are real and similar over $\mathbb{C}$, they must be also similar over $\mathbb{R}[66]$, that is, there must exist a real invertible matrix $\mathbf{T}$ such that $\mathbf{A}=\mathbf{T}^{-1} \boldsymbol{\Lambda} \mathbf{T}$. On the other hand if $\mathbf{A}$ has the form given in (28), it is diagonalizable since $\boldsymbol{\Lambda}$ is diagonalizable.

Proof of Theorem 4. Substituting the definition of a Householder transformation into the cost function in (31), here termed $\Phi(\mathbf{v})$, leads with simple algebraic manipulations to $\Phi(\mathbf{v})=\left(\mathbf{v}^{T} \mathbf{v}-1\right) \mathbf{v}^{T} \mathbf{v}-\mathbf{v}^{T} \mathbf{D} \mathbf{v}+$ const. Minimizing $\Phi(\mathbf{v})$ subject to $\mathbf{v}^{T} \mathbf{v}=1$ is therefore equivalent to maximising $\Phi_{1}(\mathbf{v})=\mathbf{v}^{T} \mathbf{D v}$ under the same constraint. The new cost function can be further expressed as

$$
\Phi_{1}(\mathbf{v})=\frac{1}{2}\left(\mathbf{v}^{T} \mathbf{D} \mathbf{v}+\mathbf{v}^{T} \mathbf{D}^{T} \mathbf{v}\right)=\frac{1}{2} \mathbf{v}^{T}\left(\mathbf{D}+\mathbf{D}^{T}\right) \mathbf{v}
$$

and the unit norm vector which maximise it, is therefore the singular vector which corresponds to the largest singular value of $\mathbf{D}+\mathbf{D}^{T}$

Proof of Theorem 5. The property that $\mathbf{S}$ scatters energy from each node equally among all other nodes requires that all off-diagonal elements are identical, and thus that $\mathbf{A}$ has the form

$$
\mathbf{A}=\left[\begin{array}{cccc}
a_{1} & a_{0} & \cdots & a_{0} \\
a_{0} & a_{2} & \cdots & a_{0} \\
\cdots & \cdots & \cdots & \cdots \\
a_{0} & a_{0} & \cdots & a_{K}
\end{array}\right]
$$

Orthogonality of $\mathbf{A}$ requires that $a_{0}, \ldots, a_{K}$ satisfy

$$
\left\{\begin{array}{ll}
a_{i}^{2}+(K-1) a_{0}^{2}=1 & i=1, \ldots, K \\
a_{0}\left(a_{i}+a_{j}\right)+(K-2) a_{0}^{2}=0 & \forall i \neq j
\end{array} .\right.
$$

The first constraint can be written as $a_{i}= \pm \sqrt{1-(K-1) a_{0}^{2}}$, which implies that all diagonal elements are identical in magnitude. The second constraint implies that if $a_{0} \neq 0$ (the solution $a_{0}=0$ is ignored since it does not scatter energy), the diagonal elements have also the same sign. Solving (44) with $a_{1}=\cdots=a_{K}$ yields $a_{1}= \pm(2-K) / K$ and $a_{0}= \pm 2 / K$, thus proving the theorem.

\section{ACKNOWLEDGMENTS}

The authors would like to thank Jonathan Abel and Geoffrey Robinson for their help and support.

\section{REFERENCES}

[1] V. Välimäki, J. D. Parker, L. Savioja, J. O. Smith, and J. S. Abel, "Fifty years of artificial reverberation," IEEE Trans. on Audio, Speech and Language Process., vol. 20, no. 5, pp. 1421-1448, Jul. 2012.

[2] M. R. Schroeder and B. F. Logan, "Colorless artificial reverberation," IRE Trans. on Audio, vol. AU-9, pp. 209-214, Nov. 1961.

[3] M. R. Schroeder, "Natural sounding artificial reverberation," J. Audio Eng. Soc., vol. 10, no. 3, pp. 219-233, 1962.
[4] M. Gerzon, "Unitary (energy-preserving) multichannel networks with feedback," IET Electronics Letters, vol. 12, no. 11, pp. 278-279, 1976.

[5] J. Stautner and M. Puckette, "Designing multichannel reverberators," Computer Music J., vol. 6, no. 1, pp. 52-65, 1982.

[6] J.-M. Jot and A. Chaigne, "Digital delay networks for designing artificial reverberators," presented at the 104th Audio Eng. Soc. Conv., Preprint \#3030, Paris, France, Feb. 1991.

[7] J. Huopaniemi, V. Valimaki, M. Karjalainen, and R. Vaananen, "Efficient and parametric reverberator for room acoustics modeling," in Proc. of the 23rd Int. Comput. Music Conf., Thessaloniki, Greece, September 1997, pp. 200-203.

[8] L. Dahl and J.-M. Jot, "A reverberator based on absorbent all-pass filters," in Proc. 3rd Int. Conf. on Digital Audio Effects (DAFx-O0), Verona, Italy, 2000.

[9] K.-S. Lee, J. S. Abel, V. Välimäki, T. Stilson, and D. P. Berners, "The switched convolution reverberator," J. Audio Eng. Soc., vol. 60, no. 4, pp. 227-236, 2012.

[10] D. Rocchesso and J. O. Smith, "Circulant and elliptic feedback delay networks for artificial reverberation," IEEE Trans. on Speech and Audio Process., vol. 5, no. 1, pp. 51-63, January 1997.

[11] J. O. Smith, "A new approach to digital reverberation using closed waveguide networks," in Proc. 11th Int. Comput. Music Conf., Burnaby, Canada, 1985, pp. 47-53.

[12] W. Koontz, "Multiport acoustic models with applications in audio signal processing," J. Audio Eng. Soc., vol. 61, no. 10, pp. 727-736, 2013.

[13] M. Vorlander, Auralization: fundamentals of acoustics, modelling, simulation, algorithms and acoustic virtual reality. Springer Verlag, 2008.

[14] L. Savioja, J. Backman, A. Järvinen, and T. Takala, "Waveguide mesh method for low-frequency simulation of room acoustics," in 15th International Congress on Acoustics (ICA), Jun. 1995, pp. 637-640.

[15] J. B. Allen and D. A. Berkley, "Image method for efficiently simulating small-room acoustics," J. Acoust. Soc. Am., vol. 65, no. 4, pp. 943-950, Apr. 1979.

[16] J. Borish, "Extension of the image model to arbitrary polyhedra," $J$. Acoust. Soc. Am., vol. 75, no. 6, pp. 1827-1836, 1984.

[17] S. Bilbao and J. O. Smith, III, "Finite difference schemes and digital waveguide networks for the wave equation: Stability, passivity, and numerical dispersion," IEEE Trans. on Speech and Audio Processing, vol. 11, no. 3, pp. 255-266, May 2003.

[18] D. Murphy, A. Kelloniemi, J. Mullen, and S. Shelley, "Acoustic modeling using the digital waveguide mesh," IEEE Signal Process. Mag., vol. 24, no. 2, pp. 55-66, 2007.

[19] M. Karjalainen and C. Erkut, "Digital waveguides versus finite difference structures: Equivalence and mixed modeling," EURASIP Journal on Applied Signal Processing, vol. 2004, pp. 978-989, 2004.

[20] M. Zyda, "From visual simulation to virtual reality to games," Computer, vol. 38, no. 9, pp. 25-32, September 2005.

[21] M. A. Gerzon, "Ambisonics in multichannel broadcasting and video," $J$. Audio Eng. Soc., vol. 33, no. 11, pp. 859-871, November 1985.

[22] M. M. Boone, U. Horbach, and W. P. J. Bruijn, "Spatial sound-field reproduction by wave-field synthesis," J. Audio Eng. Soc., vol. 43, no. 12, pp. 1003-1012, December 1995.

[23] H. Møller, "Fundamentals of binaural technology," Appl. Acoust., vol. 36, no. 3-4, pp. 171-218, 1991.

[24] SMPTE 2036-2-2008: Ultra High Definition Television - Audio Characteristics and Audio Channel Mapping for Program Production. Society of Motion Picture and Television Engineers, 2008.

[25] D. Rocchesso, "The ball within the box: a sound-processing metaphor," Computer Music Journal, pp. 47-57, 1995.

[26] M. Karjalainen, P. Huang, and J. O. Smith, "Digital Waveguide Networks for Room Response Modeling and Synthesis," presented at the 118th Audio Eng. Soc. Conv., Preprint \# 6394, Barcelona, Spain, May 2005.

[27] E. De Sena, H. Hacıhabiboğlu, and Z. Cvetković, "Scattering delay network: an interactive reverberator for computer games," in Proc. 41 st Audio Eng. Soc. Int. Conf.: Audio for Games, London, UK, Feb. 2011.

[28] H. Hacihabiboglu, E. De Sena, and Z. Cvetkovic, "Frequency-domain scattering delay networks for simulating room acoustics in virtual environments," in Proc. Signal-Image Technology and Internet-Based Systems (SITIS), November/December 2011, pp. 180-187.

[29] P. Huang and J. S. Abel, "Aspects of reverberation echo density," presented at the 123rd Audio Eng. Soc. Conv., Preprint \#7163, New York, USA, Oct. 2007

[30] W. G. Gardner, "Reverberation algorithms," in Applications of Digital Signal Processing to Audio and Acoustics, M. Kahrs and K. Brandenburg, Eds. Kluwer Academic, 1998, pp. 85-131. 
[31] J.-M. Jot, "An analysis/synthesis approach to real-time artificial reverberation," in Proc. IEEE Int. Conf. on Acoust. Speech and Signal Process. (ICASSP-92), vol. 2, 1992, pp. 221-224.

[32] C. Faller and F. Menzer, "Unitary Matrix Design for Diffuse Jot Reverberators," in Proc. 128th Conv. Audio Eng. Soc. , Preprint \# 7984, London, UK, 2010.

[33] J.-M. Jot, "Efficient models for reverberation and distance rendering in computer music and virtual audio reality," in Proc. 23rd Int. Comput. Music Conf., 1997.

[34] J. O. Smith, Physical audio signal processing. W3K Publishing, 2010, URL: https: //ccrma.stanford.edu/ jos/pasp/.

[35] G. Evangelista and F. Eckerholm, "Player-instrument interaction models for digital waveguide synthesis of guitar: Touch and collisions," IEEE Trans. on Audio, Speech and Language Process., vol. 18, no. 4, pp. 822-832, 2010.

[36] J. Sheaffer, M. van Walstijn, and B. Fazenda, "Physical and numerical constraints in source modeling for finite difference simulation of room acoustics," J. Acoust. Soc. Am., vol. 135, no. 1, pp. 251-261, 2014.

[37] D. T. Murphy, A. Southern, and L. Savioja, "Source excitation strategies for obtaining impulse responses in finite difference time domain room acoustics simulation," Applied Acoustics, vol. 82, pp. 6-14, 2014.

[38] D. T. Murphy, "Digital waveguide mesh topologies in room acoustics modelling," Ph.D. dissertation, University of York, 2000.

[39] S. D. Bilbao, Wave and scattering methods for numerical simulation. Wiley, 2004.

[40] A. Kelloniemi, V. Välimäki, and L. Savioja, "Simulation of room acoustics using 2-D digital waveguide meshes," in Proc. IEEE Int. Conf. Acoust., Speech and Signal Process. (ICASSP-06), vol. 5, Toulouse, France, May 2006.

[41] H. Hacıhabiboğlu, B. Günel, and Z. Cvetković, "Simulation of directional microphones in digital waveguide mesh-based models of room acoustics," IEEE Trans. on Audio, Speech and Language Process., vol. 18, no. 2, pp. 213-223, February 2010.

[42] J. C. Strikwerda, Finite Difference Schemes and Partial Differential Equations, 2nd ed. Philadelphia, PA, USA: Society for Industrial and Applied Mathematics, 2004.

[43] L. Savioja and V. Välimäki, "Interpolated rectangular 3-D digital waveguide mesh algorithms with frequency warping," IEEE Trans. on Speech and Audio Process., vol. 11, no. 6, pp. 783-790, November 2004.

[44] H. Kuttruff, Room Acoustics, 4th ed. SPON Press, 2000.

[45] F. Rumsey, Spatial audio. Focal Press, 2001.

[46] J. Huopaniemi, L. Savioja, and M. Karjalainen, "Modeling of reflections and air absorption in acoustical spaces a digital filter design approach," in Proc. IEEE Workshop on Appl. of Signal Process. to Audio and Acoust. (WASPAA'97), New Paltz, NY, USA, October 1997.

[47] J. A. Moorer, "About this reverberation business," Computer Music Journal, vol. 3, no. 2, pp. 13-28, 1979.

[48] J. O. Smith and D. Rocchesso, "Aspects of digital waveguide networks for acoustic modeling applications," Web published at http://ccrma.stanford.edu/jos/wgj, 1997.

[49] Z. Zhang, "A flexible new technique for camera calibration," IEEE Trans. Pattern Anal. Mach. Intell., vol. 22, no. 11, pp. 1330-1334, 2000.

[50] M. Poletti, "A unified theory of horizontal holographic sound systems," J. Audio Eng. Soc., vol. 48, no. 12, pp. 1155-1182, December 2000.

[51] V. Pulkki, "Virtual sound source positioning using vector-base amplitude panning," J. Audio Eng. Soc., vol. 45, no. 6, pp. 456-466, June 1997.

[52] J. D. Johnston and Y. H. Lam, "Perceptual soundfield reconstruction," presented at the 109th Audio Eng. Soc. Conv., Preprint \#2399, Los Angeles, CA, USA, September 2000.

[53] E. De Sena, H. Hacıhabiboğlu, and Z. Cvetković, "Analysis and design of multichannel systems for perceptual sound field reconstruction," IEEE Trans. on Audio, Speech and Language Process., vol. 21, no. 8, pp. 1653-1665, August 2013.

[54] E. A. Lehmann and A. M. Johansson, "Diffuse reverberation model for efficient image-source simulation of room impulse responses," IEEE Trans. on Audio, Speech and Language Process., vol. 18, no. 6, pp. 1429-1439, 2010.

[55] A. Oppenheim, R. Schafer, and J. Buck, Discrete-time signal processing, 2nd ed. Prentice Hall, 1999.

[56] Recomm. BR.265-9, Operating practices for the international exchange of programmes** on film for television use. International Telecommunications Union, 2004.

[57] A. Primavera, S. Cecchi, L. Romoli, P. Peretti, and F. Piazza, "A low latency implementation of a non-uniform partitioned convolution algorithm for room acoustic simulation," Signal, Image and Video Processing, vol. 8, no. 5, pp. 985-994, 2014.
[58] J. Atkins, A. Strauss, and C. Zhang, "Approximate convolution using partitioned truncated singular value decomposition filtering for binaural rendering," in Proc. IEEE Int. Conf. on Acoust. Speech and Signal Process. (ICASSP-13), Vancouver, Canada, May 2013, pp. 176-180.

[59] B. Holm-Rasmussen, H. Lehtonen, and V. Välimäki, "A new reverberator based on variable sparsity convolution," in Proc. 16th Int. Conf. on Digital Audio Effects (DAFx-13), Maynooth, Ireland, September 2013.

[60] W. C. Sabine, "Reverberation," in Collected papers on acoustics. Harvard university press, 1922

[61] C. F. Eyring, "Reverberation time in "dead" rooms," J. Acoust. Soc. Am., vol. 1 , p. $168,1930$.

[62] Various, Standard 3382. Acoustics-Measurement of the reverberation time of rooms with reference to other acoustical parameters, 1997.

[63] M. Vetterli and J. Kovačević, Wavelets and subband coding. Prentice Hall PTR, 1995, vol. 152.

[64] M. Schroeder and K. Kuttruff, "On frequency response curves in rooms. comparison of experimental, theoretical, and monte carlo results for the average frequency spacing between maxima," J. Acoust. Soc. Am., vol. 34, no. 1, pp. 76-80, 1962.

[65] [On-line]. Available: http://www.desena.org/sdn.

[66] L. Hogben, Handbook of linear algebra. CRC Press, 2006.

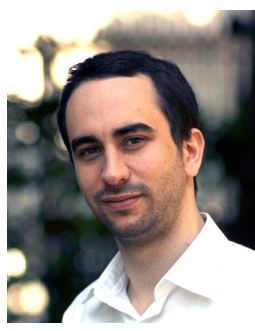

Enzo De Sena (S'11-M'14) received the B.Sc. in 2007 and M.Sc. cum laude in 2009, both from the Università degli Studi di Napoli "Federico II" in Telecommunication Engineering. In 2013, he received the Ph.D. degree from King's College London in Electronic Engineering. He is currently a Postdoctoral Research Fellow at KU Leuven. From 2012 to 2013 he was a Teaching Fellow at King's College London. From 2013 to 2015 he was a Marie Curie Fellow in the "Dereverberation and Reverberation of Audio, Music, and Speech" ITN at KU Leuven. He previously collaborated with the Network Research Lab at UCLA (20072009), and he was a Visiting Researcher at the Center for Computer Research in Music and Acoustics at Stanford University (2013) and at the Signal and Information Processing section at Aalborg University (2014-2015). His current research interests include room acoustics modelling, multichannel audio systems, microphone beamforming and binaural modelling. He is a member of IEEE, EURASIP, and the Acoustical Society of America.

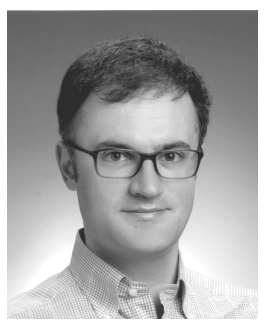

Hüseyin Hachhabiboğlu (S'96-M'00-SM'12) received the B.Sc. (honors) degree from the Middle East Technical University (METU), Ankara, Turkey, in 2000, the M.Sc. degree from the University of Bristol, Bristol, U.K., in 2001, both in electrical and electronic engineering, and the Ph.D. degree in computer science from Queen's University Belfast, Belfast, U.K., in 2004. He held research positions at University of Surrey, Guildford, U.K. (2004-2008) and King's College London, London, U.K. (20082011). Currently, he is an Associate Professor and Head of Department of Modelling and Simulation at Graduate School of Informatics, Middle East Technical University, Ankara, Turkey. His research interests include audio signal processing, room acoustics, multichannel audio systems, psychoacoustics of spatial hearing, microphone arrays, and game audio. Dr. Hacıhabiboğlu is a member of the IEEE Signal Processing Society, Audio Engineering Society (AES), Turkish Acoustics Society (TAD), and the European Acoustics Association (EAA). 


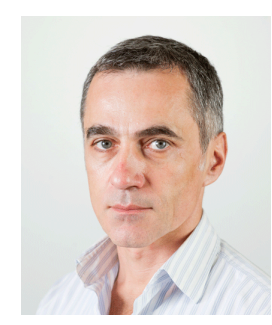

Zoran Cvetković (SM’04) is Professor of Signal Processing at King's College London. He received his Dipl. Ing. and Mag. degrees from the University of Belgrade, Yugoslavia, the M.Phil. from Columbia University, and the Ph.D. in electrical engineering from the University of California, Berkeley. He held research positions at EPFL, Lausanne, Switzerland (1996), and at Harvard University (2002-04). Between 1997 and 2002 he was a member of the technical staff of AT\&T Shannon Laboratory. His research interests are in the broad area of signal processing, ranging from theoretical aspects of signal analysis to applications in audio and speech technology, and neuroscience. From 2005 to 2008 he served as an Associate Editor of IEEE Transactions on Signal Processing.

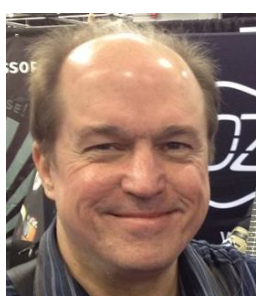

Julius O. Smith received the B.S.E.E. degree from Rice University, Houston, TX, in 1975 (Control, Circuits, and Communication). He received the M.S. and $\mathrm{Ph} . \mathrm{D}$. degrees in E.E. from Stanford University, Stanford, CA, in 1978 and 1983, respectively. His $\mathrm{Ph} . \mathrm{D}$. research was devoted to improved methods for digital filter design and system identification applied to music and audio systems. From 1975 to 1977 he worked in the Signal Processing Department at ESL, Sunnyvale, CA, on systems for digital communications. From 1982 to 1986 he was with the Adaptive Systems Department at Systems Control Technology, Palo Alto, CA, where he worked in the areas of adaptive filtering and spectral estimation. From 1986 to 1991 he was employed at NeXT Computer, Inc., responsible for sound, music, and signal processing software for the NeXT computer workstation. After NeXT, he became an Associate Professor at the Center for Computer Research in Music and Acoustics (CCRMA) at Stanford, teaching courses and pursuing research related to signal processing techniques applied to music and audio systems. Continuing this work, he is presently Professor of Music and (by courtesy) Electrical Engineering at Stanford University. For more information, see http://ccrma.stanford.edu//jos/ 